\title{
Non-standard interactions and the CP phase measurements in neutrino oscillations at low energies
}

\author{
Shao-Feng Ge and Alexei Yu. Smirnov \\ Max-Planck-Institut für Kernphysik, \\ Saupfercheckweg 1, Heidelberg 69117, Germany \\ E-mail: gesf02@gmail.com, smirnov@mpi-hd.mpg.de
}

AbStRACT: We study the effects of non-standard interactions (NSI) and the genuine CP phase $\delta_{D}$ in neutrino oscillations at low, $E_{\nu} \lesssim 1 \mathrm{GeV}$, and very low, $E_{\nu} \lesssim 0.1 \mathrm{GeV}$, energies. For experimental setup with baseline and neutrino energy tuned to the first 1-3 oscillation maximum, we develop a simple analytic formalism to show the effects of NSI. The vacuum mimicking and its violation as well as the use of the separation basis play a central role in our formalism. The NSI corrections that affect the CP phase measurement mainly come from the violation of vacuum mimicking as well as from the corrections to the 1-3 mixing angle and mass-squared difference. We find that the total NSI correction to the $\nu_{\mu}-\nu_{e}$ probability $P_{\mu e}$ can reach $20 \%-30 \%(1 \sigma)$ at T2 $(\mathrm{H}) \mathrm{K}$. Correspondingly, the correction to the $\mathrm{CP}$ phase can be as large as $50^{\circ}$ and hence significantly deteriorates the $\mathrm{CP}$ sensitivity at $\mathrm{T} 2(\mathrm{H}) \mathrm{K}$. The proposed TNT2K experiment, a combination of $\mathrm{T} 2(\mathrm{H}) \mathrm{K}$ and the short baseline experiment $\mu \mathrm{Kam}$ that uses the Super-K/Hyper-K detector at Kamioka to measure the oscillation of the antineutrinos from muon decay at rest ( $\mu \mathrm{DAR})$, can substantially reduce the degeneracy between NSI and the genuine CP phase $\delta_{D}$ to provide high CP sensitivity. The NSI correction to $P_{\mu e}$ is only $2 \%(1 \sigma)$ for $\mu$ DAR neutrinos.

KeYwords: CP violation, Neutrino Physics

ARXIV EPRINT: 1607.08513 


\section{Contents}

1 Introduction 1

2 Neutrino oscillation in matter at low energies 3

2.1 Generalities and physics setup 3

2.2 Oscillation probability 5

$\begin{array}{lll}2.3 & \text { On vacuum mimicking } & 7\end{array}$

3 Standard interaction and vacuum mimicking $\quad 9$

$\begin{array}{ll}3.1 \text { Oscillation parameters } & 9\end{array}$

$\begin{array}{ll}3.2 & \text { The oscillation probability } \\ & 10\end{array}$

4 Effects of non-standard interactions $\quad 12$

$\begin{array}{lll}4.1 & \text { Oscillation parameters in the presence of NSI } & 12\end{array}$

$\begin{array}{ll}4.2 \text { Matter corrections and the violation of vacuum mimicking } & 15\end{array}$

$\begin{array}{ll}4.3 & \text { Corrections to the total oscillation probability } 20\end{array}$

5 CP measurement in the presence of non-standard interactions $\quad 22$

5.1 The CP sensitivity at T2K in the presence of NSI 24

5.2 Improving the CP sensitivity with neutrinos from $\mu$ DAR 25

$\begin{array}{llr}6 & \text { Conclusions } & 28\end{array}$

\section{Introduction}

The determination of the Dirac CP phase, $\delta_{D}$, and searches for new (non-standard) neutrino interactions [1-3] are among the main objectives of present-day neutrino physics (see $[4,5]$ for reviews). There are certain connections between the two unknowns:

- NSI can be additional source of CP violation on top of the PMNS matrix [6, 7].

- The effects of NSI and CP violation are entangled in oscillation experiments, thus creating the $\delta_{D}$-NSI degeneracy problem $[7,8]$.

Within the standard $3 \nu$ paradigm (no NSI, no sterile neutrinos, etc.), the global fit of oscillation data gives more than $2 \sigma$ indication of $\mathrm{CP}$ violation with the best-fit value $\delta_{D} \approx 3 \pi / 2$. This indication follows mainly from the results of $\mathrm{T} 2 \mathrm{~K}[9]$ and $\mathrm{NO} \nu \mathrm{A}$ [10] in combination with the results of reactor and some other experiments. The confirmation of this hint and measurement of the CP phase are associated to future experiments with neutrinos of different origins. The current T2K experiment can be extended to T2K-II [11, 12] by upgrading the flux and eventually to T2HK with a much larger Hyper-K detector [13], 
or even T2KK/T2KO [14-21] with longer baseline. The DUNE [22-24] experiment should provide precision measurement of $\delta_{D}$. In addition to accelerator experiments, the CP phase $\delta_{D}$ can also have large observable effect in the oscillation of atmospheric neutrinos below $1 \mathrm{GeV}$. This can be explored using the future possible upgrades of PINGU and ORCA detectors with $3 \sim 4$ times denser instrumentation [25]. At even lower energy, $E_{\nu} \lesssim 0.1 \mathrm{GeV}$, one can also employ neutrinos from the muon decay at rest ( $\mu \mathrm{DAR}$ ) in proposed experiments like DAE $\delta$ ALUS [26] and CI-ADS [27], or in-flight in experiments like MOMENT $[28,29]$ to measure $\delta_{D}$. Measuring the antineutrino oscillation $\bar{\nu}_{\mu} \rightarrow \bar{\nu}_{e}$ from the $\mu \mathrm{DAR}$ source with the detector of JUNO/RENO-50, which is originally designed to establish the neutrino mass hierarchy, can also determine the CP phase [30].

Interpretation of the present results on $\mathrm{CP}$ violation can be affected by the possible presence of non-standard interactions [31] or sterile neutrinos [32-34]. The effects of NSI substantially reduce the sensitivity to $\delta_{D}$ at the ongoing $\mathrm{T} 2 \mathrm{~K}$ and $\mathrm{NO} \nu \mathrm{A}$ experiments [31, $35,36]$, and the future long-baseline experiments, in particular, DUNE [31, 35-43]. So, the problem is to disentangle the genuine CP violation and the NSI effects in a way that can guarantee high sensitivity to the $\mathrm{CP}$ phase. To achieve this, one can use the fact that the effects of NSI, in particular the effects on CP violation, are proportional to the neutrino energy $E_{\nu}$. To disentangle the genuine CP phase from NSI, one can use two or more experiments with different neutrino energies. The effects of NSI can be as large as $O(1)$ at accelerator neutrino experiments with $E_{\nu}>500 \mathrm{MeV}$ like DUNE. The neutrino spectrum from the MOMENT source peaks around $(200 \sim 300) \mathrm{MeV}$ [28], being 10 times smaller than the peak energy at DUNE. Hence, a combination of the future MOMENT and DUNE results could reduce the degeneracy between $\delta_{D}$ and the NSI parameters [40]. Still, the NSI effects at the MOMENT energy are large. Rescaled from the estimation for T2K, the relative correction of NSI to the oscillation probability $P_{\mu e}$ is roughly $10 \%$ at MOMENT. For neutrinos from $\mu$ DAR with energies $\sim(30-50) \mathrm{MeV}$ the NSI corrections are only $2 \%$.

Various possible experiments with $\mu \mathrm{DAR}$ sources have been explored. In particular, it was proposed [44] to use the Super-K detector (and later Hyper-K) to detect the antineutrino oscillation $\bar{\nu}_{\mu} \rightarrow \bar{\nu}_{e}$ from $\mu \mathrm{DAR}$ source to Kamioka (dubbed by $\mu \mathrm{Kam}$, which can be either $\mu \mathrm{SK}$ with the Super-K detector or $\mu \mathrm{HK}$ with the Hyper-K detector). The combination of $\mathrm{T} 2 \mathrm{~K}$ running solely in the neutrino $(\nu \mathrm{T} 2 \mathrm{~K})$ and $\mu \mathrm{SK}$ in the antineutrino mode, referred as TNT2K, provides even higher sensitivity to the CP phase $\delta_{D}$ [44].

In most papers, the NSI effects have been computed numerically although several analytical studies can be found in $[6,35,45,46]$. These earlier studies provide analytical expressions of the oscillation probabilities in the $\bar{\nu}_{e} \rightarrow \bar{\nu}_{e}[45,46], \nu_{e} \rightarrow \nu_{\mu}[6,46], \nu_{\mu} \rightarrow$ $\nu_{e}[35,45,46]$, and $\nu_{\mu} \rightarrow \nu_{\mu}[45,46]$ channels. In particular, [46] provides the probability formula for all oscillation channels. In this paper, we present an analytical formalism which allows us to analyze the effects of NSI in a simple way. The vacuum mimicking ${ }^{1}$ in the 1-2 sector and its violation plays a central role in our formalism. We show that NSI can induce large correction via the violation of vacuum mimicking and apply our results to the CP measurement at TNT2K.

\footnotetext{
${ }^{1}$ The effect of vacuum mimicking was noticed in [1, 47, 48], explained in [49], further studied in [50-52] and dubbed as such in [53].
} 
The paper is organized as follows. In section 2 we present a general formalism of matter effects and discuss the role of vacuum mimicking in the sector of 1-2 mass splitting and mixing. We then apply this formalism to the case of standard interactions in section 3 and non-standard interactions in section 4 . The vacuum mimicking can be significantly violated by NSI and the Dirac CP phase $\delta_{D}$ can receive $\mathcal{O}(1)$ correction. In section 5 we explore the sensitivity of $\mathrm{T} 2 \mathrm{~K}$ and $\mu \mathrm{SK}$ experiments to the genuine $\mathrm{CP}$ phase in the presence of NSI. Especially, the improvement on the sensitivity due to the $\mu$ SK component of TNT2K is shown. Our conclusions are given in section 6 .

\section{Neutrino oscillation in matter at low energies}

\subsection{Generalities and physics setup}

In the flavor basis, the Hamiltonian $\mathcal{H}$ that describes the neutrino propagation is a sum of the vacuum term and the matrix of matter potential $\mathbb{V}$ :

$$
\mathcal{H} \equiv \frac{1}{2 E_{\nu}} U_{\mathrm{PMNS}}\left(\begin{array}{lll}
0 & & \\
& \Delta m_{21}^{2} & \\
& & \Delta m_{31}^{2}
\end{array}\right) U_{\mathrm{PMNS}}^{\dagger}+\mathbb{V},
$$

where $\Delta m_{21}^{2} \equiv m_{2}^{2}-m_{1}^{2}, \Delta m_{31}^{2} \equiv m_{3}^{2}-m_{1}^{2}$, and $E_{\nu}$ is the neutrino energy. The matrix of matter potential $\mathbb{V}$ in general has contributions from both standard and non-standard interactions. For the mixing matrix in vacuum $U_{\text {PMNS }}$ we use the standard parametrization

$$
U_{\mathrm{PMNS}} \equiv U_{23}\left(\theta_{23}\right) \Gamma_{\delta_{D}} U_{13}\left(\theta_{13}\right) \Gamma_{\delta_{D}}^{\dagger} U_{12}\left(\theta_{12}\right), \quad \Gamma_{\delta_{D}}=\operatorname{diag}\left(1,1, e^{i \delta_{D}}\right)
$$

Here $\theta_{i j}$ are the vacuum mixing angles and $\delta_{D}$ is the Dirac CP phase. In the case of standard interaction only, the matrix of matter potential takes the form as

$$
\mathbb{V}=\operatorname{diag}\{V, 0,0\} \quad \text { and } \quad V=\sqrt{2} G_{F} n_{e}
$$

where $G_{F}$ is the Fermi coupling and $n_{e}$ is the electron number density. For the standard interaction with a constant density $3 \mathrm{~g} / \mathrm{cm}^{3}$ of isotopically neutral matter and $\sin ^{2} \theta_{12} \approx$ 0.31 , the resonance neutrino energy due to the 1-2 mass splitting equals

$$
E_{R}=\cos 2 \theta_{12} \frac{\Delta m_{21}^{2}}{2 V}=122 \mathrm{MeV} .
$$

At low energy, $E_{\nu} \lesssim 1 \mathrm{GeV}$, the three mass-energy scales $\left(\Delta m_{21}^{2}, \Delta m_{31}^{2}, 2 E_{\nu} V\right)$ in the Hamiltonian (2.1) satisfy the following conditions:

$$
\frac{\Delta m_{21}^{2}}{2 E_{\nu}} \sim V \ll \frac{\Delta m_{31}^{2}}{2 E_{\nu}} .
$$

This allows us to introduce two small parameters

$$
r_{\Delta} \equiv \frac{\Delta m_{21}^{2}}{\Delta m_{31}^{2}} \approx 0.03, \quad x_{31} \equiv \frac{2 E V}{\Delta m_{31}^{2}}=0.01\left(\frac{E_{\nu}}{100 \mathrm{MeV}}\right)
$$


which will be used for perturbative diagonalization of the Hamiltonian. For typical energies $E_{\nu}=50 \mathrm{MeV}$ at $\mu \mathrm{DAR}$ and $E_{\nu}=600 \mathrm{MeV}$ at T2K, we obtain $x_{31}=5 \cdot 10^{-3}$ and $x_{31}=0.06$, respectively.

At low energies the matter corrections to the 1-3 mixing angle and mass splitting are small. It is more convenient to consider the neutrino oscillation in a different basis $\nu^{\prime}$ that is related to the flavor basis $\nu_{f}$ as, $[54,55]$,

$$
\nu_{f}=\mathcal{R} \nu^{\prime}
$$

where

$$
\mathcal{R} \equiv U_{23}\left(\theta_{23}\right) \Gamma_{\delta} U_{13}\left(\theta_{13}\right)
$$

The matrix $\mathcal{R}$ depends only on the vacuum mixing angles $\left(\theta_{23}, \theta_{13}\right)$ and the phase $\delta_{D}$. Using (2.1) and (2.7), we obtain the Hamiltonian in the $\nu^{\prime}$ basis

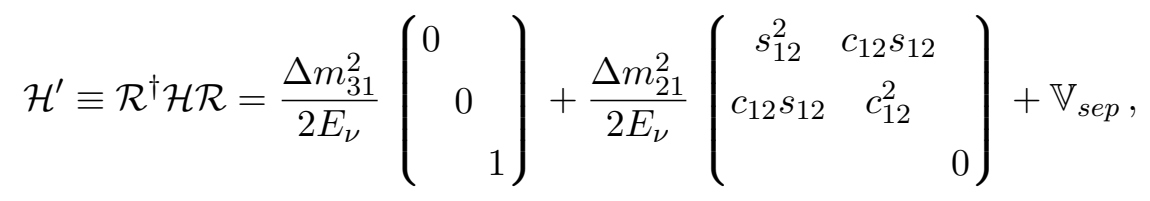

where

$$
\mathbb{V}_{\text {sep }} \equiv U_{13}^{T} \Gamma_{\delta}^{\dagger} U_{23}^{T} \mathbb{V} U_{23} \Gamma_{\delta} U_{13}
$$

and we use the notation $\left(c_{i j}, s_{i j}\right) \equiv\left(\cos \theta_{i j}, \sin \theta_{i j}\right)$. In this basis, the first term with the largest mass scale $\Delta m_{31}^{2}$ is diagonal and separated from the 1-2 sector in vacuum. The second term in (2.8) depends on the vacuum parameters of the 1-2 sector only while the last term is the matter term that provides all other (in particular 1-3) mixings. For convenience, we call this basis the separation basis. It is related to the usual propagation basis [56-58] by an additional rotation $\Gamma_{\delta} U_{13}\left(\theta_{13}\right)[54,55]$.

Let us introduce a unitary mixing matrix $U^{\prime}$ to diagonalize the Hamiltonian $\mathcal{H}^{\prime}(2.8)$ :

$$
U^{\prime \dagger} \mathcal{H}^{\prime} U^{\prime}=\operatorname{diag}\left(H_{1}, H_{2}, H_{3}\right)
$$

where $H_{i}$ are the eigenvalues of $\mathcal{H}^{\prime}$. Then the total mixing matrix in matter, $U^{m}$, which connects the flavor states and the eigenstates of the Hamiltonian $\mathcal{H}, \nu_{f}=U^{m} \nu^{m}$, is given by the product

$$
U^{m}=\mathcal{R} U^{\prime}
$$

In (2.11), the vacuum and matter parts are factorized. Without matter effect, we obtain $U^{\prime}=U_{12}\left(\theta_{12}\right)$.

In matter with constant density, solving the evolution equation is straightforward. For the eigenstates of the Hamiltonian, $\nu^{m}$, we obtain according to (2.10) the evolution matrix

$$
S^{d}=\left(\begin{array}{lll}
1 & & \\
& e^{-i \Delta H_{21} L} & \\
& & e^{-i \Delta H_{31} L}
\end{array}\right)
$$


where $\Delta H_{i j} \equiv H_{i}-H_{j}$ and $L$ is the distance (baseline). Then the matrix of amplitude in the flavor basis is

$$
S^{f}=U^{m} S^{d} U^{m \dagger},
$$

and the $\nu_{\alpha} \rightarrow \nu_{\beta}(\alpha, \beta=e, \mu, \tau)$ oscillation probability is given by $P_{\alpha \beta} \equiv\left|S_{\beta \alpha}^{f}\right|^{2}$.

In what follows we will elaborate on the analytical description of the neutrino oscillation effects for the following physics setup:

(i) Neutrino energies are from a few tenths to a few hundreds of $\mathrm{MeV}$.

(ii) The baselines are about half of the oscillation length for the 1-3 mass splitting.

$$
L \sim \frac{1}{2} l_{31}=\frac{2 \pi E_{\nu}}{\Delta m_{31}^{2}} .
$$

Correspondingly, the (half) oscillation phase equals $\phi_{31} \approx \pi / 2$.

(iii) The phase associated with the $2-1$ splitting is small:

$$
\phi_{21}=r_{\Delta} \phi_{31} \sim \frac{\pi r_{\Delta}}{2} \sim 5 \cdot 10^{-2} \quad\left(\sim 3^{\circ}\right) .
$$

Also the matter phase

$$
\phi_{V}=V L \sim \phi_{31} r_{\Delta} \cos 2 \theta_{12} \frac{E_{\nu}}{E_{R}}
$$

is small. The setup is realized in accelerator experiments such as $\mathrm{T} 2(\mathrm{H}) \mathrm{K}, \mathrm{NO} \nu \mathrm{A}$, MOMENT, and experiments based on the K-meson, pion and muon decays at rest: KDAR, $\mu \mathrm{DAR}$, etc. [59].

\subsection{Oscillation probability}

We consider the $\nu_{\mu} \rightarrow \nu_{e}$ transition in matter. Using (2.12) and (2.13), the probability $P_{\mu e}$ can be generally presented as

$$
P_{\mu e}=\left|2 U_{e 2}^{m *} U_{\mu 2}^{m} \sin \phi_{21}^{m}+2 U_{e 3}^{m *} U_{\mu 3}^{m} \sin \phi_{31}^{m} e^{-i \phi_{32}^{m}}\right|^{2},
$$

where $U_{\alpha j}^{m}$ is the $\alpha j$-element of the mixing matrix $U^{m}$ in matter, and the half-oscillation phases equal

$$
\phi_{21}^{m}=\frac{1}{2} \Delta H_{21} L, \quad \phi_{31}^{m}=\frac{1}{2} \Delta H_{31} L, \quad \phi_{32}^{m}=\phi_{31}^{m}-\phi_{21}^{m} .
$$

We can rewrite the probability $P_{\mu e}$ using the explicit expressions of $U_{\alpha j}^{m}$ in the standard parametrization. In particular, the first term in (2.17) becomes

$$
2 c_{13}^{m} s_{12}^{m}\left(c_{12}^{m} c_{23}^{m}-s_{13}^{m} s_{12}^{m} s_{23}^{m} e^{i \delta_{D}^{m}}\right) \sin \phi_{21}^{m},
$$

where $\left(c_{i j}^{m}, s_{i j}^{m}\right) \equiv\left(\cos \theta_{i j}^{m}, \sin \theta_{i j}^{m}\right)$. It is convenient to combine the second term in (2.19) with the second term in (2.17) to get

$$
P_{\mu e} \equiv\left|A_{e \mu}^{S}+A_{e \mu}^{A}\right|^{2} .
$$


Here, we have introduced the "solar" amplitude

$$
A_{e \mu}^{S}=c_{13}^{m} c_{23}^{m} A_{12}^{m}
$$

with

$$
A_{12}^{m} \equiv \sin 2 \theta_{12}^{m} \sin \phi_{21}^{m},
$$

and the "atmospheric" amplitude

$$
A_{e \mu}^{A} \equiv s_{23}^{m} \sin 2 \theta_{13}^{m} e^{i \delta_{D}^{m}}\left[\sin \phi_{31}^{m} e^{-i \phi_{32}^{m}}-s_{12}^{m 2} \sin \phi_{21}^{m}\right] .
$$

The atmospheric amplitude contains the term with oscillation phase related to the small 2-1 mass splitting and it can be rewritten as

$$
A_{e \mu}^{A}=s_{23}^{m} A_{13}^{m} e^{i\left(\delta_{D}^{m}-\phi_{32}^{m}\right)}\left[1-\left(s_{12}^{m}\right)^{2} e^{i \phi_{32}^{m}} \frac{\sin \phi_{21}^{m}}{\sin \phi_{31}^{m}}\right]
$$

where

$$
A_{13}^{m} \equiv \sin 2 \theta_{13}^{m} \sin \phi_{31}^{m} .
$$

The amplitudes $A_{13}^{m}$ and $A_{12}^{m}$ coincide with the standard $2 \nu$ oscillation amplitudes.

The 2-1 splitting correction to the atmospheric amplitude (expression in the brackets of (2.24)) can be parametrized as

$$
\left[1-y e^{i \phi_{32}^{m}}\right] \equiv \kappa e^{-i \phi_{\kappa}}
$$

where

$$
y \equiv s_{12}^{m 2}\left(\frac{\sin \phi_{21}^{m}}{\sin \phi_{31}^{m}}\right) .
$$

For the experimental setup under consideration $y \approx s_{12}^{m 2} r_{\Delta}$. Below the 1-2 resonance $\left(E_{\nu}<E_{R}\right)$ we have $s_{12}^{m 2} \approx s_{12}^{2}=0.31$, in the resonance $s_{12}^{m 2}=0.5$, and $s_{12}^{m 2}$ approaches 1 with $E_{\nu}$ above the resonance. So, typically $y=(1-3) \cdot 10^{-2}$ is a small quantity. The quantities in (2.26) equal

$$
\begin{aligned}
\kappa & =\sqrt{1-2 y \cos \phi_{32}^{m}+y^{2}} \approx 1-y \cos \phi_{32}^{m}, \\
\tan \phi_{\kappa} & =\frac{y \sin \phi_{32}^{m}}{1-y \cos \phi_{32}^{m}} \approx y \sin \phi_{32}^{m} .
\end{aligned}
$$

Since $y \ll 1$, the phase $\phi_{\kappa}$ is much smaller than $\phi_{32}^{m}$.

Thus, the total probability (2.17) can be written as

$$
P_{\mu e}=\left|c_{13}^{m} c_{23}^{m} A_{12}^{m}+s_{23}^{m} A_{13}^{m} \kappa e^{i\left(\delta_{D}^{m}-\phi_{32}^{m}-\phi_{\kappa}\right)}\right|^{2} .
$$

Here $\phi_{\kappa}=\phi_{\kappa}\left(\phi_{32}^{m}\right)$ is small correction to the oscillation phase $\phi_{32}^{m}$. If $\kappa=1$, eq. (2.29) reproduces the standard expression for $P_{\mu e}$.

In the case of standard interactions, the vacuum mimicking for the 1-2 amplitude is realized due to smallness of the $2-1$ phase $\phi_{21}^{m} \ll 1$, [1, 47-53]

$$
A_{12}^{m} \equiv \sin 2 \theta_{12}^{m} \sin \phi_{21}^{m} \approx \sin 2 \theta_{12} \sin \phi_{21}=A_{12}
$$


For $\phi_{21}^{m} \ll 1,(2.30)$ gives $A_{12}^{m} \simeq \sin 2 \theta_{12} \frac{\Delta m_{21}^{2} L}{2 E_{\nu}}$. The condition $\phi_{21}^{m} \ll 1$ for vacuum mimicking is fulfilled in the majority of existing and proposed experiments.

In general, vacuum mimicking is broken in the presence of NSI [49]. Introducing parameter

$$
r_{V} \equiv \frac{A_{12}^{m}}{A_{12}}
$$

to quantify the deviation from vacuum mimicking, we can rewrite the ptobability $P_{\mu e}$ as

$$
P_{\mu e} \approx\left|c_{13}^{m} c_{23}^{m} r_{V} A_{12}+s_{23}^{m} A_{13}^{m} \kappa e^{i\left(\delta_{D}^{m}-\phi_{32}^{m}-\phi_{\kappa}\right)}\right|^{2} .
$$

This parametrization of the probability $P_{\mu e}$ is convenient for understanding the matter effects, including both standard and non-standard interactions.

The probability $P_{\mu e}$ in $(2.32)$ can also be rewritten as sum of the atmospheric, solar and interference terms:

$$
P_{\mu e} \approx P_{\mu e}^{A}+P_{\mu e}^{S}+P_{\mu e}^{I} \cos \left(\delta_{D}^{m}-\phi_{32}^{m}-\phi_{\kappa}\right)
$$

Here

$$
\begin{aligned}
& P_{\mu e}^{A}=\left|A_{\mu e}^{A}\right|^{2} \equiv \kappa^{2} s_{23}^{m 2} \sin ^{2} 2 \theta_{13}^{m} \sin ^{2} \phi_{31}^{m}, \\
& P_{\mu e}^{S}=\left|A_{\mu e}^{S}\right|^{2} \approx r_{V}^{2} c_{23}^{m 2} c_{13}^{m 2} \sin ^{2} 2 \theta_{12} \sin ^{2} \phi_{21}, \\
& P_{\mu e}^{I} \equiv \kappa r_{V} \sin 2 \theta_{23}^{m} c_{13}^{m} \sin 2 \theta_{13}^{m} \sin \phi_{31}^{m} \sin 2 \theta_{12} \sin \phi_{21} .
\end{aligned}
$$

For constant density, the problem then reduces to finding the mixing angles and mass splittings in matter. The exact vacuum mimicking corresponds to $r_{V}=1$. If in addition $\kappa=$ 1 and $\phi_{\kappa}=0$, eqs. (2.33)-(2.36) reproduce the usual approximate expression for $P_{\mu e}[60]$.

It is easy to see that the 1-2 correction to the atmospheric amplitude

$$
\kappa-1 \propto \sin ^{2} \theta_{12}^{m} \sin \phi_{21}^{m}=A \frac{\Delta m_{21}^{2} L}{4 E_{\nu}}
$$

does not show vacuum mimicking and has strong dependence on the matter potential $V$. For $V \rightarrow 0: A \rightarrow \sin ^{2} \theta_{12}$, in the resonance $A \rightarrow \sin 2 \theta_{12}$ and with further increase of $V$ the coefficient $A$ increases as $\propto V$.

\subsection{On vacuum mimicking}

Let us present vacuum mimicking in a general form for both standard and non-standard interactions. Consider for simplicity the $2 \nu$ Hamiltonian with moduli of the off-diagonal elements $|\overline{\mathcal{H}}|$ and difference of the eigenvalues $\Delta \mathcal{H}$. The mixing angle is given by

$$
\sin 2 \theta_{12}^{m}=\frac{2|\overline{\mathcal{H}}|}{\Delta \mathcal{H}}
$$

and the (half) oscillation phase equals

$$
\phi_{21}^{m}=\frac{1}{2} \Delta \mathcal{H} L
$$


Then

$$
A_{21}^{m} \approx \sin 2 \theta_{12}^{m} \phi_{21}^{m}=\frac{2|\overline{\mathcal{H}}|}{\Delta \mathcal{H}} \frac{1}{2} \Delta \mathcal{H} L=|\overline{\mathcal{H}}| L .
$$

So, the oscillation amplitude $A_{21}^{m}$ in the first order is given by the off-diagonal element of the $2 \nu$ Hamiltonian multiplied by distance. Since for standard interaction the matter potential appears only in the diagonal elements of the Hamiltonian in the flavor basis, $\overline{\mathcal{H}}=\overline{\mathcal{H}}_{V}$, we obtain $A_{21}^{m}=A_{21}$. The deviation from vacuum mimicking appears when the off-diagonal element $\overline{\mathcal{H}}$ depends on matter potential.

Even for the standard interactions, matter effect appears in $A_{12}^{m}$ when $\sin \phi_{21}^{m}$ is expanded to higher order,

$$
A_{12}^{m} \equiv \sin 2 \theta_{12} \frac{\Delta m_{21}^{2} L}{2 E_{\nu}}\left[1-\frac{1}{6}\left(\frac{\Delta H_{21} L}{2}\right)^{2}\right] .
$$

For the vacuum amplitude $A_{12}$ we have the same expression with substitution $\Delta H_{21} \rightarrow$ $\Delta m_{21}^{2} / 2 E_{\nu}$. Then the ratio of the matter to vacuum amplitudes equals

$$
r_{V}=1+\frac{1}{3}\left(\phi_{21}\right)^{2} \cos ^{2} 2 \theta_{12} c_{13}^{2}\left(1-\frac{c_{13}^{2}}{2} \frac{E}{E_{R}}\right)
$$

Using (2.15) we can estimate the matter corrections as

$$
r_{V}-1 \approx \frac{1}{3}\left(\frac{\pi r_{\Delta}}{2}\right)^{2} \cos ^{2} 2 \theta_{12}\left(1-\frac{E}{2 E_{R}}\right) \approx 10^{-4}
$$

According to (2.43), tn addition to the small oscillation phase squared, the correction $r_{V}-1$ is suppressed by $\cos ^{2} 2 \theta_{12} / 3 \approx 0.03$. Note that $r_{V}-1$ is positive for neutrinos because $\Delta H_{21}<\Delta m_{21}^{2} / 2 E_{\nu}$ and increases with energy. For antineutrinos it is negative.

One comment is in order. Recently there was a discussion on why the formulas for the $3 \nu$-oscillation probabilities in matter derived for $E \gg E_{21}^{R}$ (i.e., far above the 1-2 resonance) work well at $E \sim E_{21}^{R}$ [61]. Rather complicated explanation has been proposed in [62]. In fact, the reason is simple. For small values of the 2-1 phase, which is true at all proposed long-baseline experiments, the vacuum mimicking is realized. In spite of large matter corrections to the oscillation phase and mixing angle, the corrections cancel with each other in the oscillation probabilities. The same expression for the oscillation probability applies at all energies as long as the matter phase is small and the presence of the 1-2 resonance or not is irrelevant.

Corrections due to $\kappa(2.28 \mathrm{a})$ and (2.37) depend on the 1-2 sector parameters, $\kappa=$ $\kappa\left(\theta_{21}, \Delta_{21}, V\right)$, and break vacuum breaking. In the $3 \nu$ case the vacuum mimicking does not work exactly even for the standard interaction. As can be seen from (2.33) the matter effect associated with the 1-2 splitting, $\phi_{21}^{m}$, appears in the phase of the interference term. To realize the vacuum mimicking, one needs to reduce $3 \nu$ evolution to $2 \nu$ evolution associated with small mass splitting. In the $3 \nu$ case, the violation of vacuum mimicking can be induced even by the diagonal elements of the matter potential matrix (see below). 


\section{Standard interaction and vacuum mimicking}

\subsection{Oscillation parameters}

Using the expression (2.2) of $\mathbb{V}$ for the standard interactions in the flavor basis, we obtain the matrix of matter potential in the separation basis,

$$
\mathbb{V}_{\text {sep }}=U_{13}^{T} \mathbb{V} U_{13}=V\left(\begin{array}{ccc}
c_{13}^{2} & & c_{13} s_{13} \\
& 0 & \\
c_{13} s_{13} & & s_{13}^{2}
\end{array}\right)
$$

Consequently, the total Hamiltonian (2.8) can be written explicitly as

$$
\mathcal{H}^{\prime}=\frac{\Delta m_{31}^{2}}{2 E_{\nu}}\left(\begin{array}{cc}
0 & \\
& 0 \\
& \\
&
\end{array}\right)+\frac{\Delta m_{21}^{2}}{2 E_{\nu}}\left(\begin{array}{ccc}
s_{12}^{2} & c_{12} s_{12} \\
c_{12} s_{12} & c_{12}^{2} & \\
& & 0
\end{array}\right)+V\left(\begin{array}{ccc}
c_{13}^{2} & & c_{13} s_{13} \\
& 0 & \\
& & \\
c_{13} s_{13} & & s_{13}^{2}
\end{array}\right)
$$

Here the 1-3 mixing (being proportional to $\theta_{13}$ ) is generated by matter potential.

An additional 1-3 rotation

$$
\delta \theta_{13} \approx \tan \delta \theta_{13} \approx s_{13} c_{13} \frac{2 E_{\nu} V}{\Delta m_{31}^{2}}=s_{13} c_{13} x_{31}
$$

eliminates the 1-3 and 3-1 elements of the Hamiltonian (3.2). This rotation, in turn, generates non-zero 2-3 and 3-2 elements which have next order of smallness and can be neglected. Consequently, $\theta_{23}^{m} \approx \theta_{23}$ (see [55] for details).

After the rotation (3.3) the third state decouples and for the rest of the system we obtain the effective $2 \nu$ Hamiltonian

$$
\mathcal{H}_{2 \nu}^{\prime} \approx \frac{\Delta m_{21}^{2}}{2 E_{\nu}}\left(\begin{array}{cc}
s_{12}^{2}+c_{13}^{2} x_{21} & c_{12} s_{12} \\
c_{12} s_{12} & c_{12}^{2}
\end{array}\right),
$$

where

$$
x_{21} \equiv \frac{2 E_{\nu} V}{\Delta m_{21}^{2}}=0.28\left(\frac{E_{\nu}}{100 \mathrm{MeV}}\right) .
$$

For typical energies of $\mu \mathrm{DAR}$ and $\mathrm{T} 2 \mathrm{~K}$, we have $x_{21}=0.14$ and $x_{21}=1.5$. The correction to the 1-1 element generated by decoupling is of the order $r_{\Delta} s_{13}^{2}$, and therefore has been neglected in (3.4). Notice that the Hamiltonian (3.4) can be obtained by blockdiagonalization (3.2) and decoupling of the third state.

The diagonalization of (3.4) gives the effective mass splitting

$$
\Delta H_{21}=\frac{\Delta m_{21}^{2}}{2 E} \sqrt{\sin ^{2} 2 \theta_{12}+\left(\cos 2 \theta_{12}-c_{13}^{2} x_{21}\right)^{2}},
$$

and the 1-2 mixing angle in matter

$$
\sin 2 \theta_{12}^{m}=\frac{\Delta m_{21}^{2}}{2 E \Delta H_{21}} \sin 2 \theta_{12}
$$


The 1-3 splitting (upper sign) and the 2-3 spitting (lower sign) are

$$
\Delta H_{31}\left(\Delta H_{32}\right) \approx \frac{\Delta m_{31}^{2}}{2 E}-\frac{1}{2}\left[\frac{\Delta m_{21}^{2}}{2 E} \mp \Delta H_{21}+\left(c_{13}^{2}-2 s_{13}^{2}\right) V\right] .
$$

Thus, the diagonalization matrix in the separation basis is given by

$$
U^{\prime}=U_{13}\left(\delta \theta_{13}\right) U_{12}\left(\theta_{12}^{m}\right)
$$

and the total mixing matrix in matter becomes

$$
U^{m}=\mathcal{R} U^{\prime}=U_{23}\left(\theta_{23}\right) \Gamma_{\delta_{D}} U_{13}\left(\theta_{13}^{m}\right) U_{12}\left(\theta_{12}^{m}\right),
$$

where

$$
\theta_{13}^{m}=\theta_{13}+\delta \theta_{13} .
$$

The matter correction to the CP phase is absent: $\delta_{D}^{m}=\delta_{D}$.

\subsection{The oscillation probability}

The probability of $\nu_{\mu} \rightarrow \nu_{e}$ transition is given in (2.33)-(2.36). Taking $r_{V} \approx 1, c_{23}^{m} \approx c_{23}$, $s_{23}^{m} \approx s_{23}$ and $\delta_{D}^{m}=\delta_{D}$, it becomes

$$
\begin{aligned}
P_{\mu e}= & \kappa^{2} s_{23}^{2} \sin ^{2} 2 \theta_{13}^{m} \sin ^{2} \phi_{31}^{m}+c_{23}^{2} c_{13}^{m 2} \sin ^{2} 2 \theta_{12} \sin ^{2} \phi_{21} \\
& +\kappa \sin 2 \theta_{23} c_{13}^{m} \sin 2 \theta_{13}^{m} \sin \phi_{31}^{m} \sin 2 \theta_{12} \sin \phi_{21} \cos \left(\delta_{D}-\phi_{32}^{m}-\phi_{\kappa}\right) .
\end{aligned}
$$

The correction to the atmospheric amplitude given by $(\kappa-1) \sim r_{\Delta}$ can also be neglected. Then for $\kappa=1$ and $\phi_{\kappa}=0$, (3.12) reproduces the commonly used formula of $P_{\mu e}$ [60]. The oscillation parameters in matter $\theta_{13}^{m}, \phi_{31}^{m}$, and $\phi_{32}^{m}$ that enter this formula have been obtained in eqs. (3.6), (3.7), (3.8), (3.11).

In figure 1 (left panel) we show the dependence of the probability $P_{\mu e}$ on $E_{\nu} / L$ for the T2K setup and different values of $\delta_{D}$. At the first oscillation maximum, $E_{\nu} / L \approx 1.8$, we have $P_{\mu e}(0)=P_{\mu e}(\pi)$ and $P_{\mu e}( \pm \pi / 2)=(1 \pm 1 / 3) P_{\mu e}(0)$ where the minus sign corresponds to the presently favored $\delta_{D}=3 \pi / 2$. The spread of the values of $P_{\mu e}\left(\delta_{D}\right)$ with varying $\delta_{D}$ is more than $60 \%$.

According to (2.36) $P_{\mu e}^{A} \propto s_{13}^{2}, P_{\mu e}^{S}$ is suppressed by $\sin ^{2} \phi_{21} \propto r_{\Delta}^{2}$, and $P_{\mu e}^{I} \propto s_{13} r_{\Delta}$. So, the following hierarchy

$$
\begin{aligned}
& \frac{P_{\mu e}^{S}}{P_{\mu e}^{A}} \approx \sin ^{2} 2 \theta_{12} \frac{\phi_{21}^{2}}{4 s_{13}^{2}} \approx \frac{2}{9}\left(\frac{\pi r_{\Delta}}{2 s_{13}}\right)^{2} \approx 2 \%, \\
& \frac{P_{\mu e}^{I}}{P_{\mu e}^{A}} \approx \frac{\sin 2 \theta_{12}}{s_{13}} \phi_{21} \approx \frac{\pi r_{\Delta}}{2 s_{13}} \approx 30 \%,
\end{aligned}
$$

is realized between the three components at the first oscillation maximum. Roughly, $P_{\mu e}^{A}$ : $P_{\mu e}^{I}: P_{\mu e}^{S}=50: 15: 1$ which holds also above the resonance (see figure 1) but changes significantly with decrease of energy: at $E_{\nu} / L \approx 1.2$ we have $P_{\mu e}^{A}=P_{\mu e}^{I}$ and $P_{\mu e}^{S}=P_{\mu e}^{A} / 4$. Similar relations between the different components of $P_{\mu e}$ are also realized at $\mu \mathrm{SK}$. 

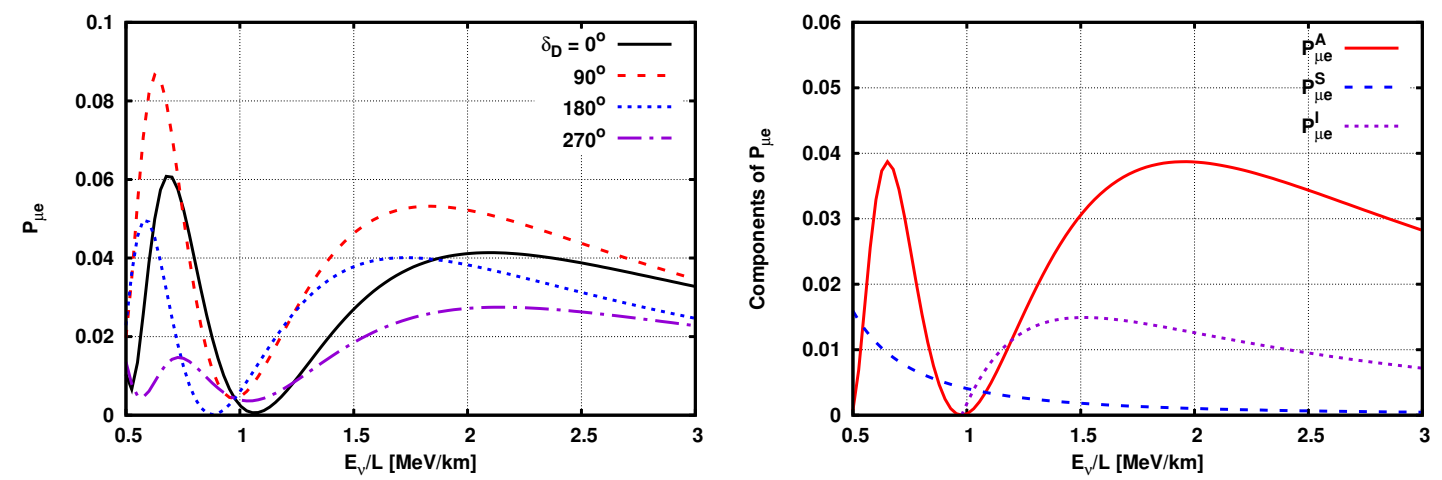

Figure 1. The oscillation probability $P_{\mu e}$ as a function of $E_{\nu} / L$ for different values of the CP phase $\delta_{D}$ (left). Different components of $P_{\mu e}\left(P_{\mu e}^{A}, P_{\mu e}^{I}, P_{\mu e}^{S}\right)$ as functions of $E_{\nu} / L$ for $\delta_{C P}=3 \pi / 2$. We use the $\mathrm{T} 2(\mathrm{H}) \mathrm{K}$ baseline $L=295 \mathrm{~km}$.

The matter correction to the whole oscillation probability $P_{\mu e}$ mainly comes from the angle $\theta_{13}^{m}$ and the phase $\phi_{31}^{m}$. Using $P_{\mu e}^{A}$ only we find

$$
\begin{aligned}
V \frac{\partial P_{\mu e}^{A}}{\partial V} & \approx \frac{1}{2} s_{23}^{2} \sin ^{2} 2 \theta_{13} \sin \phi_{31}\left[4 \sin \phi_{31} \frac{2 E_{\nu} V}{\Delta m_{31}^{2}}-c_{13}^{2} \cos \phi_{31} V L\right] \\
& \approx s_{23}^{2} \sin ^{2} 2 \theta_{13} \sin ^{2} \phi_{31} \frac{4 E_{\nu} V}{\Delta m_{31}^{2}}\left[1-c_{13}^{2} \cot \phi_{31} \frac{\phi_{31}}{2}\right] \\
& \approx \sin ^{2} 2 \theta_{13} x_{31}\left(1-0.5 c_{13}^{2} \phi_{31} \cot \phi_{31}\right) .
\end{aligned}
$$

The first term in (3.14) comes from $\theta_{13}^{m}$ while the second from $\phi_{31}^{m}$. At the first oscillation maximum, the second term is suppressed by $\cot \phi_{31} \approx 0$. However, if the neutrino energy spectrum is wide enough, $\cos \phi_{31}$ can become sizable out of the peak, as shown in figure 2 . According to figure $2, P_{\mu e}$ and $P_{\mu e}^{I}$ at $\mathrm{T} 2 \mathrm{~K}$ and $\mu \mathrm{SK}$ are very similar when expressed as functions of $E_{\nu} / L$. At the first oscillation maximum $P_{\mu e}^{I} / P_{\mu e} \approx 0.3$ and the matter effect increases with energy as

$$
P_{\mu e}-P_{\mu e}^{\mathrm{vac}} \propto E_{\nu}
$$

according to (3.14). Therefore the matter effect at $\mu \mathrm{SK}$ is typically 12 times smaller than at T2K. With respect to the interference $(\mathrm{CP})$ term, the matter effect is about $(25-30) \%$ at $\mathrm{T} 2 \mathrm{~K}$ and $(2.0-2.2) \%$ at $\mu \mathrm{SK}$.

For standard interactions, the matter corrections mainly come from the 1-3 mixing $s_{13}^{m}$, being $\sim 13 \%$ at $\mathrm{T} 2 \mathrm{~K}$ and $\sim 1 \%$ at $\mu \mathrm{DAR}$, at the first oscillation peak and for $\delta_{D}=3 \pi / 2$. The matter effect from the 1-2 sector is strongly suppressed by the vacuum mimicking, producing $\mathcal{O}\left(r_{\Delta}\right)$ correction to the atmospheric amplitude $A_{\mu e}^{A}$. The interference term is about $1 / 3$ of the total probability. 

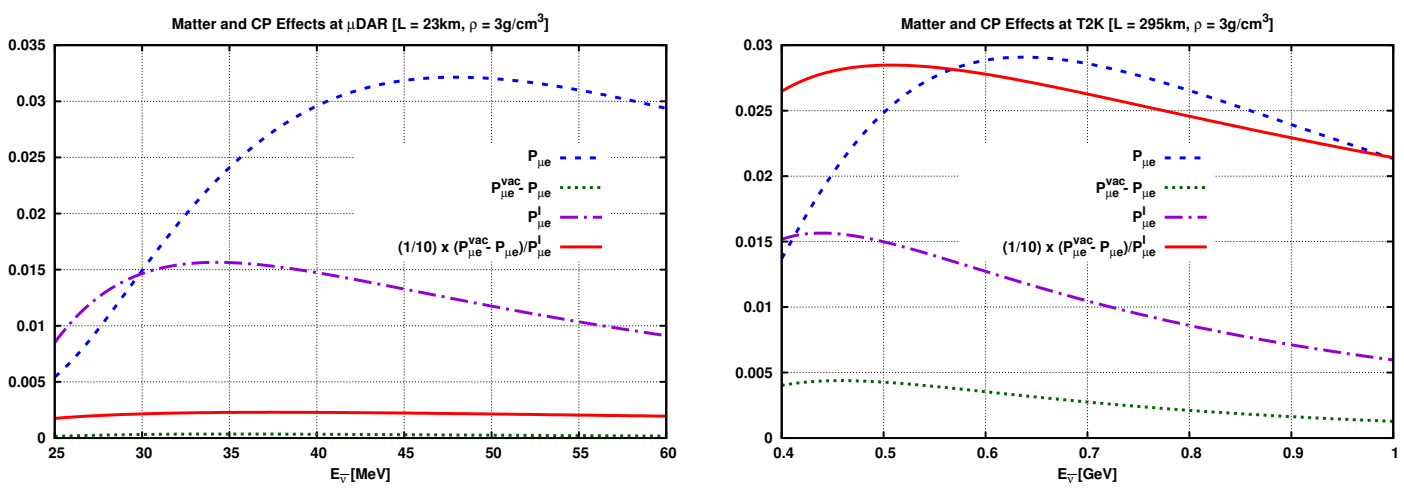

Figure 2. The matter effect on probabilities $\left(P_{\mu e}^{\mathrm{vac}}-P_{\mu e}\right)$ and the $\mathrm{CP}$ term coefficient $P_{\mu e}^{I}$ at $\mu \mathrm{DAR}$ (left) and $\mathrm{T} 2(\mathrm{H}) \mathrm{K}$ (right) for $\delta_{D}=3 \pi / 2$.

\section{Effects of non-standard interactions}

\subsection{Oscillation parameters in the presence of NSI}

In the presence of NSI, the matter potential matrix $\mathbb{V}$ in the flavor basis can be parametrized as

$$
\mathbb{V}=\left(\begin{array}{lll}
V & & \\
& 0 & \\
& &
\end{array}\right)+V\left(\begin{array}{lll}
\epsilon_{e e} & \epsilon_{e \mu} & \epsilon_{e \tau} \\
\epsilon_{e \mu}^{*} & \epsilon_{\mu \mu} & \epsilon_{\mu \tau} \\
\epsilon_{e \tau}^{*} & \epsilon_{\mu \tau}^{*} & \epsilon_{\tau \tau}
\end{array}\right)
$$

(We do not separate here scattering on different components of matter: electrons, $\mathrm{u}-\mathrm{and}$ d- quarks, summing up the effect.) The $1 \sigma$ constraints on the NSI parameters $\epsilon_{\alpha \beta}$ from the global fit [63] are

$$
\begin{aligned}
& \left|\epsilon_{e \mu}\right|<0.16, \\
& \left|\epsilon_{e \tau}\right|<0.26, \\
& \left|\epsilon_{\mu \tau}\right|<0.02, \\
& -0.018<\epsilon_{\tau \tau}-\epsilon_{\mu \mu}<0.054, \\
& 0<\epsilon_{e e}-\epsilon_{\mu \mu}<0.93 .
\end{aligned}
$$

The hierarchy among these constraints can be described by powers of small parameter $\lambda=0.15$ (notice that $s_{13} \approx \lambda$ ):

$$
\epsilon_{e e}-\epsilon_{\mu \mu}<\mathcal{O}(1), \quad \epsilon_{e \mu}, \quad \epsilon_{e \tau}<\mathcal{O}(\lambda), \quad \epsilon_{\mu \tau}, \quad \epsilon_{\tau \tau}-\epsilon_{\mu \mu}<\mathcal{O}\left(\lambda^{2}\right)
$$

Consequently, the matrix of matter potential in the flavor basis can have the maximal allowed $1 \sigma$ values,

$$
\mathbb{V} \sim V\left(\begin{array}{ccc}
1 & \lambda & \lambda \\
\lambda & \lambda^{2} & \lambda^{2} \\
\lambda & \lambda^{2} & \lambda^{2}
\end{array}\right)
$$

In the separation basis, the matrix of matter potentials is given by transformation of (4.1):

$$
\mathbb{V}_{\text {sep }}=U_{13}^{T} \Gamma_{\delta}^{\dagger} U_{23}^{T} \mathbb{V} U_{23} \Gamma_{\delta} U_{13}=V\left(\begin{array}{ccc}
\epsilon_{11}^{\prime}+c_{13}^{2} & \epsilon_{12}^{\prime} & \epsilon_{13}^{\prime}+s_{13} c_{13} \\
\epsilon_{12}^{\prime *} & \epsilon_{22}^{\prime} & \epsilon_{23}^{\prime} \\
\epsilon_{13}^{\prime *}+s_{13} c_{13} & \epsilon_{23}^{*} & \epsilon_{33}^{\prime}+s_{13}^{2}
\end{array}\right),
$$


where

$$
\begin{aligned}
& \epsilon_{11}^{\prime}=c_{13}^{2}\left(\epsilon_{e e}-\epsilon_{\mu \mu}\right)+s_{13}^{2}\left[c_{23}^{2}\left(\epsilon_{\tau \tau}-\epsilon_{\mu \mu}\right)+2 c_{23} s_{23} \mathbb{R}\left(\epsilon_{\mu \tau}\right)\right]-2 c_{13} s_{13} \mathbb{R}\left[\left(s_{23} \epsilon_{e \mu}+c_{23} \epsilon_{e \tau}\right) e^{i \delta_{D}}\right], \\
& \epsilon_{12}^{\prime}=c_{13}\left(c_{23} \epsilon_{e \mu}-s_{23} \epsilon_{e \tau}\right)+s_{13} e^{-i \delta_{D}}\left[c_{23} s_{23}\left(\epsilon_{\tau \tau}-\epsilon_{\mu \mu}\right)-\left(\cos 2 \theta_{23} \mathbb{R}-i \mathbb{I}\right)\left(\epsilon_{\mu \tau}\right)\right], \\
& \epsilon_{13}^{\prime}=c_{13} s_{13}\left[\left(\epsilon_{e e}-\epsilon_{\mu \mu}\right)-c_{23}^{2}\left(\epsilon_{\tau \tau}-\epsilon_{\mu \mu}\right)-2 c_{23} s_{23} \mathbb{R}\left(\epsilon_{\mu \tau}\right)\right]+\left(\cos 2 \theta_{13} \mathbb{R}+i \mathbb{I}\right)\left[\left(s_{23} \epsilon_{e \mu}+c_{23} \epsilon_{e \tau}\right) e^{i \delta_{D}}\right], \\
& \epsilon_{22}^{\prime}=-2 c_{23} s_{23} \mathbb{R}\left(\epsilon_{\mu \tau}\right)+s_{23}^{2}\left(\epsilon_{\tau \tau}-\epsilon_{\mu \mu}\right), \\
& \epsilon_{23}^{\prime}=s_{13}\left(c_{23} \epsilon_{e \mu}^{*}-s_{23} \epsilon_{e \tau}^{*}\right)-c_{13} e^{i \delta_{D}}\left[c_{23} s_{23}\left(\epsilon_{\tau \tau}-\epsilon_{\mu \mu}\right)-\left(\cos 2 \theta_{23} \mathbb{R}+i \mathbb{I}\right)\left(\epsilon_{\mu \tau}\right)\right], \\
& \epsilon_{33}^{\prime}=s_{13}^{2}\left(\epsilon_{e e}-\epsilon_{\mu \mu}\right)+c_{13}^{2}\left[c_{23}^{2}\left(\epsilon_{\tau \tau}-\epsilon_{\mu \mu}\right)+2 c_{23} s_{23} \mathbb{R}\left(\epsilon_{\mu \tau}\right)\right]+2 c_{13} s_{13} \mathbb{R}\left[\left(s_{23} \epsilon_{e \mu}+c_{23} \epsilon_{e \tau}\right) e^{i \delta_{D}}\right] .
\end{aligned}
$$

The operators $\mathbb{R}$ and $\mathbb{I}$ extract the real and imaginary parts from the attached quantities, e.g. $\mathbb{R}\left(\epsilon_{\mu \tau}\right) \equiv \operatorname{Re}\left(\epsilon_{\mu \tau}\right)$. For convenience, we have subtracted from $\mathbb{V}$ a diagonal term $\epsilon_{\mu \mu}$ proportional to the unit matrix $\mathbb{I}_{3 \times 3}$ which does not affect oscillation probabilities. Notice that the elements of $\mathbb{V}_{\text {sep }}$ in the separation basis have the same hierarchy of values as (4.4) in the flavor basis due to hierarchical values of the mixing angles $s_{13}=\mathcal{O}(\lambda)$ and $s_{23}=\mathcal{O}(1)$ in (4.5).

We can diagonalize the total Hamiltonian in the same way as in the case of standard interaction. First, block-diagonalization gives the effective $2 \nu$ Hamiltonian

$$
\mathcal{H}^{\prime}{ }_{2 \nu} \approx \frac{\Delta m_{21}^{2}}{2 E_{\nu}}\left(\begin{array}{cc}
s_{12}^{2}+x_{21}\left(c_{13}^{2}+\epsilon_{11}^{\prime}\right) & s_{12} c_{12}+x_{21} \epsilon_{12}^{\prime} \\
s_{12} c_{12}+x_{21} \epsilon_{12}^{\prime *} & c_{12}^{2}+x_{21} \epsilon_{22}^{\prime}
\end{array}\right)
$$

for the first two states. The decoupled state has the eigenvalue

$$
H_{3} \approx \frac{\Delta m_{31}^{2}}{2 E}+V\left(s_{13}^{2}+\epsilon_{33}^{\prime}\right) .
$$

In the first approximation, this block-diagonalization is equivalent to an additional 1-3 rotation

$$
\Gamma_{\delta_{3}} U_{13}\left(\delta \theta_{13}\right)
$$

which removes the 1-3 and 3-1 elements of the total Hamiltonian. Here

$$
\Gamma_{\delta_{3}} \equiv \operatorname{diag}\left(1,1, e^{i \delta_{3}}\right), \quad \delta_{3}=\operatorname{Arg}\left[s_{13} c_{13}+\epsilon_{13}^{\prime}\right],
$$

and the rotation angle in $U_{13}\left(\delta \theta_{13}\right)$ is determined approximately by

$$
\delta \theta_{13} \approx \tan \delta \theta_{13} \approx x_{31} \frac{\left|s_{13} c_{13}+\epsilon_{13}^{\prime}\right|}{1+x_{31}\left(s_{13}^{2}+\epsilon_{33}^{\prime}\right)} \approx x_{31}\left|s_{13} c_{13}+\epsilon_{13}^{\prime}\right| .
$$

Numerically, $\delta \theta_{13} \sim \mathcal{O}\left(\lambda^{3}\right)$, but the phase $\delta_{3}$ can be large, $\delta_{3}=\mathcal{O}(1)$, since $\epsilon_{13}^{\prime} \sim s_{13} \sim \lambda$.

In this approximation, we have neglected the 2-3 and 3-2 elements of the Hamiltonian. They can be eliminated by an additional $2-3$ rotation on the angle

$$
\delta \theta_{23} \approx x_{31}\left|\epsilon_{23}^{\prime}\right|
$$

which has the next order of smallness: $\delta \theta_{23}=\mathcal{O}\left(\lambda^{4}\right)$.

Diagonalization of the matrix (4.7) gives the effective mass splitting

$$
\Delta H_{21}=\frac{\Delta m_{21}^{2}}{2 E} \sqrt{\left[\cos 2 \theta_{12}-x_{21}\left(c_{13}^{2}+\epsilon_{11}^{\prime}-\epsilon_{22}^{\prime}\right)\right]^{2}+\left(\sin 2 \theta_{12}+2 x_{21} \epsilon_{12}^{\prime}\right)^{2}},
$$


and mixing $\Gamma_{\delta_{2}} U_{12}\left(\theta_{12}^{m}\right)$ where

$$
\begin{aligned}
\sin 2 \theta_{12}^{m} & =\frac{\Delta m_{21}^{2}}{2 E \Delta H_{21}}\left|\sin 2 \theta_{12}+2 x_{21} \epsilon_{12}^{\prime}\right|, \\
\Gamma_{\delta_{2}} & =\operatorname{diag}\left(1, e^{i \delta_{2}}\right), \quad \delta_{2}=-\operatorname{Arg}\left[\sin 2 \theta_{12}+2 x_{21} \epsilon_{12}^{\prime}\right] .
\end{aligned}
$$

Notice that with NSI the off-diagonal element of the Hamiltonian $\mathcal{H}_{2 \nu}^{\prime}$ are complex and so additional rephasing $\Gamma_{\delta_{2}}$ is needed. In fact, the phase $\delta_{2}$ also originates from the violation of vacuum mimicking, being the phase of the off-diagonal element $\mathcal{H}_{12}$ (or equivalently $\overline{\mathcal{H}}$ in (2.38) for the $2 \nu$ Hamiltonian).

Using the trace of the Hamiltonian (4.7) as well as the equalities (4.8) and (4.12), we find the 1-3 mass splitting

$$
\Delta H_{31} \approx \frac{\Delta m_{31}^{2}}{2 E}+V\left(s_{13}^{2}+\epsilon_{33}^{\prime}\right)-\frac{1}{2}\left[\frac{\Delta m_{21}^{2}}{2 E}-\Delta H_{21}+\left(c_{13}^{2}+\epsilon_{11}^{\prime}+\epsilon_{22}^{\prime}\right) V\right] .
$$

Combining all the rotations we obtain the total mixing matrix in matter

$$
U_{m}=\mathcal{R} U^{\prime}=U_{23}\left(\theta_{23}\right) \Gamma_{\delta_{D}} U_{13}\left(\theta_{13}\right) \Gamma_{\delta_{3}} U_{13}\left(\delta \theta_{13}\right) \Gamma_{\delta_{2}} U_{12}\left(\theta_{12}^{m}\right) .
$$

Notice that if $\delta_{3}=0$, the correction $\delta \theta_{13}$ can be combined with $\theta_{13}$ as in the standard interaction case (3.11).

To find the effective mixing angles in matter, the matrix (4.16) should be reduced to the standard parametrization. Since $\Gamma_{\delta_{2}}$ commutes with the 1-3 rotations on the left-hand side, it can be combined with $\Gamma_{\delta_{D}}$ :

$$
\Gamma_{\delta_{D}} \Gamma_{\delta_{2}}=\operatorname{diag}\left[1, e^{i \delta_{2}}, e^{i \delta_{2}}\right] \operatorname{diag}\left[1,1, e^{i\left(\delta_{D}-\delta_{2}\right)}\right] .
$$

The first matrix in (4.17) can be omitted since it commutes with the 2-3 rotations in (4.16), and therefore can be absorbed in the rephasing of the charged lepton states.

It is straightforward to show that the product of the 1-3 transformations $U_{13}\left(\theta_{13}\right) \Gamma_{\delta_{3}} U_{13}\left(\delta \theta_{13}\right)$ can be written as

$$
U_{13}\left(\theta_{13}\right) \Gamma_{\delta_{3}} U_{13}\left(\delta \theta_{13}\right)=\Gamma_{3} \Gamma_{C} \Gamma_{\alpha} U_{13}\left(\theta_{13}^{m}\right) \Gamma_{\beta} .
$$

Here

$$
\begin{aligned}
& \Gamma_{3}=\operatorname{diag}\left(e^{i \delta_{3} / 2}, 1, e^{i \delta_{3} / 2}\right), \\
& \Gamma_{C}=\operatorname{diag}\left(e^{i \phi_{C}}, 1, e^{i \phi_{C}}\right), \\
& \Gamma_{\alpha}=\operatorname{diag}\left(1,1, e^{-i\left(\phi_{S}+\phi_{C}\right)}\right), \\
& \Gamma_{\beta}=\operatorname{diag}\left(1,1, e^{i\left(\phi_{S}-\phi_{C}\right)}\right),
\end{aligned}
$$

and the phase $\phi_{S}$ is determined from

$$
\tan \phi_{S}=\tan \left(\frac{\delta_{3}}{2}\right) \frac{\sin \left(\theta_{13}-\delta \theta_{13}\right)}{\sin \left(\theta_{13}+\delta \theta_{13}\right)}
$$


and the phase $\phi_{C}$ has similar expression with substitution sin $\rightarrow \cos$ and overall minus sign. Then, the 1-3 mixing angle in matter is given by

$$
\cos \theta_{13}^{m}=\sqrt{\cos ^{2}\left(\delta_{3} / 2\right) \cos ^{2}\left(\theta_{13}+\delta \theta_{13}\right)+\sin ^{2}\left(\delta_{3} / 2\right) \cos ^{2}\left(\theta_{13}-\delta \theta_{13}\right)} .
$$

After inserting (4.18) into (4.16), we find a number of simplifications. The matrix $\Gamma_{\beta}$ commutes with the 1-2 rotation and therefore can be absorbed into the rephasing of the eigenstates of the Hamiltonian. The product $\Gamma_{3} \Gamma_{C} \Gamma_{\alpha}$ can be written as

$$
\Gamma_{3} \Gamma_{C} \Gamma_{\alpha}=\operatorname{diag}\left[e^{i\left(\delta_{3} / 2+\phi_{C}\right)}, 1,1\right] \times \operatorname{diag}\left[1,1, e^{i\left(\delta_{3} / 2-\phi_{S}\right)}\right] .
$$

The first matrix commutes with the 2-3 rotation of (4.16) and therefore can be absorbed into the rephasing of the flavor states. The second matrix can be combined with the matrix in (4.17). As a result, we obtain the standard expression for the mixing matrix

$$
U_{m}=\mathcal{R} U^{\prime}=U_{23}\left(\theta_{23}\right) \Gamma_{\delta_{D}^{m}} U_{13}\left(\theta_{13}^{m}\right) U_{12}\left(\theta_{12}^{m}\right)
$$

with

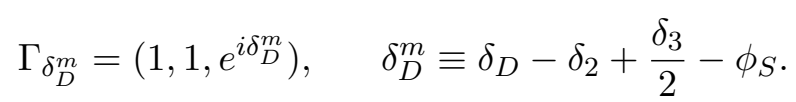

Here $\delta_{D}^{m}$ is the effective CP phase in matter which includes corrections from NSI.

The obtained expressions for the effective mixing parameters in matter can be simplified using the smallness of $\delta \theta_{13}$ (4.10). From (4.23) we find

$$
\frac{\delta_{3}}{2}-\phi_{S} \approx x_{31} c_{13}^{2}\left|1+\frac{\epsilon_{13}^{\prime}}{c_{13} s_{13}}\right| \sin \delta_{3},
$$

so that the effective $\mathrm{CP}$ phase in matter becomes

$$
\delta_{D}^{m} \approx \delta_{D}-\delta_{2}+x_{31} c_{13}^{2}\left|1+\frac{\epsilon_{13}^{\prime}}{c_{13} s_{13}}\right| \sin \delta_{3} .
$$

For the effective 1-3 mixing angle in matter we have

$$
\theta_{13}^{m} \approx \theta_{13}+\cos \delta_{3} \delta \theta_{13} .
$$

Recall that $\delta_{2}=\delta_{2}\left(\epsilon_{12}^{\prime}\right)$ is a function of $\epsilon_{12}^{\prime}$, whereas $\delta_{3}=\delta_{3}\left(\epsilon_{13}^{\prime}\right)$ is a function of $\epsilon_{13}^{\prime}$.

According to our consideration here, the matter potential (including NSI) influences the mixing angles, the CP phase, and the splittings of the eigenvalues of the Hamiltonian in rather specific ways which we will discuss in the next two subsections.

\subsection{Matter corrections and the violation of vacuum mimicking}

The matter potential influences the 1-2 mixing and splitting in a particular form that can be described with high accuracy as the violation of vacuum mimicking. The non-diagonal element of the Hamiltonian (4.7),

$$
\mathcal{H}^{\prime}{ }_{12}=s_{12} c_{12}+x_{21} \epsilon_{12}^{\prime}
$$


depends on the NSI parameter $\epsilon_{12}^{\prime}$ and therefore vacuum mimicking is broken [49] already at the lowest order, according to the discussions related to (2.40). The violation of vacuum mimicking is characterized by

$$
r_{V}=\frac{\left|\mathcal{H}^{\prime}{ }_{12}\right|}{\mathcal{H}_{12}}=\left|1+\frac{2 x_{21} \epsilon_{12}^{\prime *}}{\sin 2 \theta_{12}}\right|=\left|1+\xi_{21} \epsilon_{12}^{\prime *}\right|,
$$

where we have denoted

$$
\xi_{21} \equiv \frac{2 x_{21}}{\sin 2 \theta_{12}} .
$$

For T2K peak energy, $E_{\nu} \sim 600 \mathrm{MeV}$, the deviation $r_{V}-1 \approx 2.30 \epsilon_{12}^{\prime}$ can be as large as $\mathcal{O}(1)$. For $\mu \mathrm{DAR}\left(E_{\nu}=50 \mathrm{MeV}\right)$ we have $\left(r_{V}-1\right) \sim 0.215 \epsilon_{12}^{\prime}$. Here the deviation also scales linearly with energy. Consequently, the deviation at $\mathrm{T} 2 \mathrm{~K}$ is about 12 times larger than the one at $\mu \mathrm{DAR}$.

The violation parameter $r_{V}(2.31)$ of vacuum mimicking equals

$$
r_{V}=\sqrt{1+2 \xi_{21}\left|\epsilon_{12}^{\prime}\right| \cos \phi_{\epsilon}+\xi_{21}^{2}\left|\epsilon_{12}^{\prime}\right|^{2}}
$$

with $\phi_{\epsilon} \equiv \operatorname{Arg}\left[\epsilon_{12}^{\prime}\right]$.

There are two effects of the violation of vacuum mimicking:

1. Being attached to the solar amplitude (2.31), the phase factor $e^{i \delta_{2}}$ contributes to the phase of the interference term (2.33) directly:

$$
\delta_{D}-\delta_{2}-\phi_{32}^{m}
$$

Consequently, the $\mathrm{CP}$ phase in matter equals

$$
\delta_{D}^{m} \approx \delta_{D}-\delta_{2}
$$

in agreement with (4.29) which differs by the small correction due to the matter effect on the 1-3 mixing. Thus, the violation of vacuum mimicking gives the main correction to the $\mathrm{CP}$ phase in matter.

2. The interference term is modified by a factor $r_{V}$ and the solar term by a factor $r_{V}^{2}$. The deviation from the standard case equals

$$
r_{V}^{2}-1=\xi_{21}\left|\epsilon_{12}^{\prime}\right|\left(2 \cos \phi_{\epsilon}+\xi_{21}\left|\epsilon_{12}^{\prime}\right|\right) .
$$

Consequently, the correction vanishes when

$$
\left|\epsilon_{12}^{\prime}\right|=-\frac{2 \cos \phi_{\epsilon}}{\xi_{21}}
$$

For $\phi_{\epsilon}=0$ and $\pi$, the factor $r_{V}$ itself can be zero if

$$
\left|\epsilon_{12}^{\prime}\right|=\frac{1}{\xi_{21}}=\frac{\sin 2 \theta_{12}}{2 x_{21}}=\sin 2 \theta_{12} \frac{\Delta m_{21}^{2}}{4 E V} .
$$


Let us consider the effects of the NSI parameters $\epsilon_{e e}-\epsilon_{\mu \mu}, \epsilon_{\tau \tau}-\epsilon_{\mu \mu}, \epsilon_{e \mu}, \epsilon_{e \tau}$ and $\epsilon_{\mu \tau}$ separately. They modify the $\nu_{\mu}-\nu_{e}$ oscillation probability $P_{\mu}$ via $\epsilon_{12}^{\prime}$, see the second equation of (4.6). Notice that the largest possible NSI parameter $\epsilon_{e e}-\epsilon_{\mu \mu}$ does not contribute to $\epsilon_{12}^{\prime}$.

From (4.6) we find the parameter of the violation of vacuum mimicking in terms of the NSI parameters:

$$
r_{V}=\left|1+\frac{2 x_{21} F}{\sin 2 \theta_{12}}\right|, \quad F= \begin{cases}0 & \leq \mathcal{O}\left(\lambda^{5}\right) \\ c_{23} s_{23} s_{13} e^{i \delta_{D}}\left(\epsilon_{\tau \tau}-\epsilon_{\mu \mu}\right) & \leq \mathcal{O}\left(\lambda^{3}\right) \\ c_{23} c_{13} \epsilon_{e \mu}^{*} & \leq \mathcal{O}(\lambda) \\ -s_{23} c_{13} \epsilon_{e \tau}^{*} & \leq \mathcal{O}(\lambda) \\ -s_{13} e^{i \delta_{D}}\left(\cos 2 \theta_{23} \mathbb{R}+i \mathbb{I}\right)\left(\epsilon_{\mu \tau}\right) & \leq \mathcal{O}\left(\lambda^{3}\right) .\end{cases}
$$

The presence of several non-zero $\epsilon$ can be easily taken into account by summing up the contributions to $F$.

Let us comment on the effect of individual NSI parameter (4.39) when all others are zero.

- $\epsilon_{e e}-\epsilon_{\mu \mu} \neq 0$. Being the biggest allowed parameter, it generates $\epsilon_{11}^{\prime}, \epsilon_{13}^{\prime}$ and $\epsilon_{33}^{\prime}$ while other $\epsilon_{i j}^{\prime}$ parameters vanish. According to (4.6), the parameter $\epsilon_{11}^{\prime}$ is the diagonal element of the Hamiltonian $\mathcal{H}_{2 \nu}^{\prime}$ while $\epsilon_{13}^{\prime}$ and $\epsilon_{33}^{\prime}$ cannot contribute to $\mathcal{H}^{\prime}{ }_{2 \nu}$. Therefore, vacuum mimicking is realized: $F=0$ and $r_{V}=1$ in the leading order.

- $\epsilon_{\tau \tau}-\epsilon_{\mu \mu} \neq 0$. This parameter contributes to all $\epsilon_{i j}^{\prime}$. In $\epsilon_{12}^{\prime}$ it appears with suppression factor $s_{13}$, so that $\epsilon_{12}^{\prime}=\mathcal{O}\left(\lambda^{3}\right)$. Although $\epsilon_{\tau \tau}$ and $\epsilon_{\mu \mu}$ are the diagonal elements of the matter potential matrix in the flavor basis, they violate vacuum mimicking via $\epsilon_{12}^{\prime}$. This happens due to the $3 \nu$ mixing and large oscillation phase associated with the third state (otherwise mimicking would exist for all mass splittings [49]). We can call such a violation the induced violation of vacuum mimicking due to the $3 \nu$ mixing. The corresponding contribution to the oscillation probability is proportional to $s_{23} s_{13}$.

- $\epsilon_{e \mu} \neq 0$ produces all $\epsilon_{i j}^{\prime}$ but $\epsilon_{22}^{\prime}$. It appears in $\epsilon_{12}^{\prime}$ without suppression, and therefore provides the largest violation of vacuum mimicking. Similar statement applies for $\epsilon_{e \tau} \neq 0$.

- $\epsilon_{\mu \tau} \neq 0$ generates all $\epsilon_{i j}^{\prime}$. Being small it appears with $s_{13}$ in $\epsilon_{12}^{\prime}$.

In figure 3 we show the dependence of $r_{V}-1$ on the NSI parameters. According to figure 3 , in the $1 \sigma$ allowed interval of $\epsilon_{e \mu}$, the correction is between $\pm 45 \%$ at $\mathrm{T} 2 \mathrm{~K}$, with maxima at $\operatorname{Arg}\left[\epsilon_{e \mu}\right]=0$ and minimum at $\operatorname{Arg}\left(\epsilon_{e \mu}\right)=\pi$. At $\mu \mathrm{DAR}$, the correction is 12 times smaller: $\pm 4 \%$. For $\epsilon_{e \tau}$ the correction at T2K is slightly larger: $\pm 65 \%$ with maximum at $\operatorname{Arg}\left[\epsilon_{e \tau}\right]=\pi$.

The correction from $\epsilon_{\mu \tau}$ depends on the phase $\delta_{D}$. For $\delta_{D}=3 \pi / 2$ we have from (4.39)

$$
\xi_{21} \epsilon_{12}^{\prime}=-\xi_{21} s_{13}\left|\epsilon_{\mu \tau}\right|\left(-i \cos 2 \theta_{23} \cos \phi_{\mu \tau}+\sin \phi_{\mu \tau}\right) \approx \xi_{21} s_{13}\left|\epsilon_{\mu \tau}\right| \sin \phi_{\mu \tau}
$$



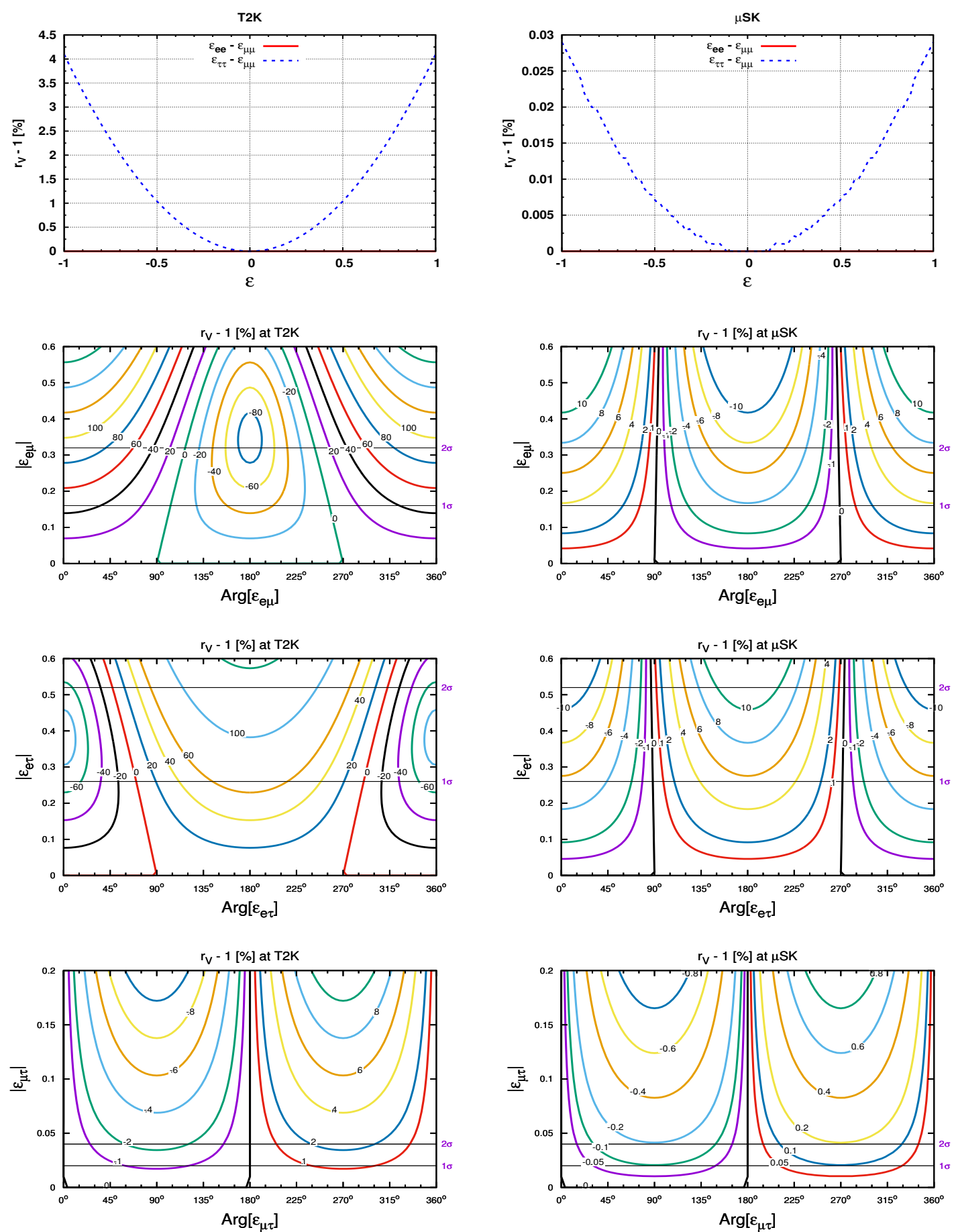

Figure 3. Dependence of the deviation from vacuum mimicking, $r_{V}-1$, on the absolute values and phases of the NSI parameters: (a) $\epsilon_{e e}-\epsilon_{\mu \mu}$ and $\epsilon_{\tau \tau}-\epsilon_{\mu \mu}$; (b) $\epsilon_{e \mu}$; (c) $\epsilon_{e \tau}$; (d) $\epsilon_{\mu \tau}$. We use $\delta_{D}=3 \pi / 2$. The left panels are for $E_{\nu}=600 \mathrm{MeV}(\mathrm{T} 2(\mathrm{H}) \mathrm{K})$ and the right panels are for $E_{\nu}=50 \mathrm{MeV}(\mu \mathrm{SK})$. The horizontal lines show $1 \sigma$ and $2 \sigma$ upper bounds on the NSI parameters. 

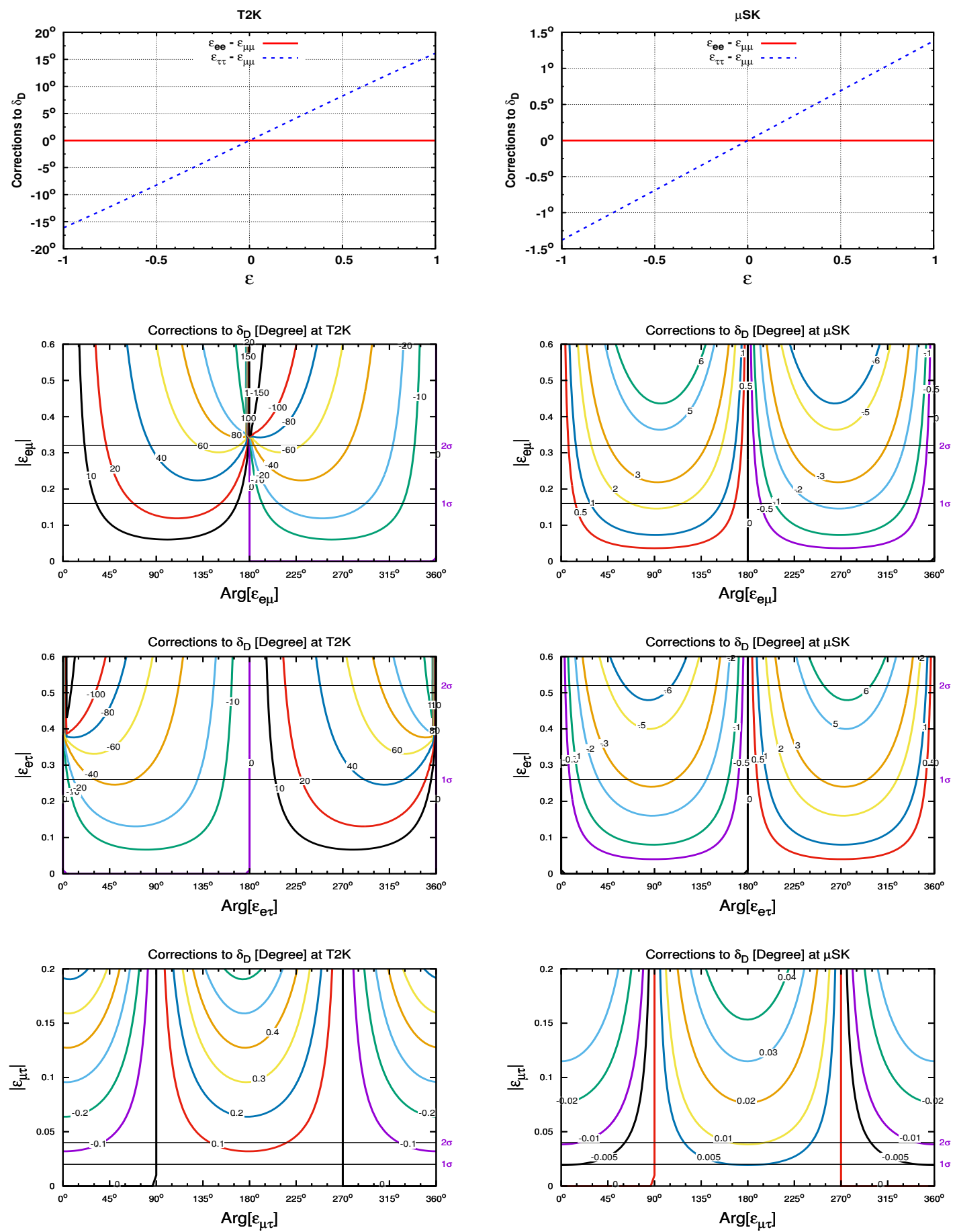

Figure 4. The same as in figure 3 for the matter correction to the leptonic Dirac CP phase $\delta_{D}^{m}-\delta_{D}=\delta_{2}$.

which gives a correction between $\pm 1.2 \%$. The parameter $\left(\epsilon_{\tau \tau}-\epsilon_{\mu \mu}\right)$ is real but its correction depends on $\delta_{D}$. For $\delta_{D}=3 \pi / 2$, we have $r_{V}=\left|1-i s_{13} s_{23} c_{23}\left(\epsilon_{\tau \tau}-\epsilon_{\mu \mu}\right)\right|$ and consequently $r_{V}-1 \approx\left[s_{13} s_{23} c_{23}\left(\epsilon_{\tau \tau}-\epsilon_{\mu \mu}\right)\right]^{2}$ which is negligible being quadratic in $s_{13}$. 
The figure 4 shows the dependence of the correction to the CP phase $\delta_{2} \approx \delta_{D}^{m}-\delta_{D}$ on the NSI parameters. The largest correction comes from $\epsilon_{e \mu}$ and $\epsilon_{e \tau}$. With the $1 \sigma$ interval of $\epsilon_{e \mu}$ we obtain $\delta_{2} \approx \pm 30^{\circ}$ at $\mathrm{T} 2 \mathrm{~K}$ and $\delta_{2}= \pm 2.2^{\circ}$ at $\mu \mathrm{DAR}$. Similar numbers are obtained for $\epsilon_{e \tau}: \delta_{2} \sim \pm 40^{\circ}$ and $\delta_{2}= \pm 3^{\circ}$, respectively. Contrary to the situation for $r_{V}$, the parameter $\epsilon_{\tau \tau}-\epsilon_{\mu \mu}$ has larger effect on $\delta_{D}^{m}-\delta_{D}$ than $\epsilon_{\mu \tau}$ does.

The attraction points in the $\epsilon_{e \mu}$ and $\epsilon_{e \tau}$ planes at $\left|\epsilon_{e \mu}\right| \approx-0.35, \phi_{e \mu}=\pi$ and $\epsilon_{e \tau} \approx 0.4$, $\phi_{e \tau}=0,2 \pi$ correspond to $r_{V}=0$ for $E_{\nu}=600 \mathrm{MeV}$, according to (4.38). (For $\mu \mathrm{DAR}$ that would appear at much larger, already excluded, values of NSI parameters.) Around the attraction points the correction $\delta_{2}$ to the CP phase $\delta_{D}$ can take any value.

From the observational point of view the deviation $r_{V}-1$ produces an additional degeneracy with $\delta_{D}$. The factor $r_{V}$ in the interference term can be absorbed in redefinition of $\delta_{D}$. Indeed, the interference term is proportional to $r_{V} \cos \left(\delta_{D}-\phi_{32}^{m}\right)$ and its variation

$$
\left(r_{V}-1\right) \cos \left(\delta_{D}-\phi_{32}^{m}\right)-\Delta \delta_{D} r_{V} \sin \left(\delta_{D}-\phi_{32}^{m}\right)
$$

vanishes when

$$
\Delta \delta_{D}=\cot \left(\delta_{D}-\phi_{32}^{m}\right) \frac{r_{V}-1}{r_{V}} .
$$

At the first oscillation maximum, $\phi_{32}^{m} \approx \pi / 2$, we obtain

$$
\Delta \delta_{D}=\tan \delta_{D} \frac{r_{V}-1}{r_{V}} .
$$

Near the maximal CP violation $\delta_{D}=3 \pi / 2$ the correction can be strongly enhanced.

\subsection{Corrections to the total oscillation probability}

The complete form of the oscillation probability $P_{\mu e}$ is given by the expression (2.33) with oscillation parameters in matter determined in section 4.1. According to (2.33) the following quantities are modified in matter: $r_{V}, \delta_{D}^{m} \approx \delta_{D}-\delta_{2}$ which are the parameters of the 1-2 sector, and $\theta_{13}^{m}, \phi_{31}^{m}, \phi_{32}^{m}$ - the parameters of the 1-3 sector. We will neglect the corrections due to $\kappa$ as well as due to the matter effect on the 2-3 mixing which is of the order $\lambda^{4}$. In what follows we will consider the NSI corrections due to the modification of these oscillation parameters in order, comparing the oscillation probabilities with $\left(P_{\mu e}^{N S I}\right)$ and without $\left(P_{\mu e}\right)$ the NSI effects:

$$
\frac{\delta P_{\mu e}}{P_{\mu e}} \equiv \frac{P_{\mu e}^{N S I}-P_{\mu e}}{P_{\mu e}}
$$

1. The violation of vacuum mimicking gives two contributions: from $r_{V}-1$ and $\delta_{2}$, both determined by $\epsilon_{12}^{\prime} \sim \lambda$. They affect the interference term $P_{\mu e}^{I}$. The violation parameter $r_{V}$ also modifies the "solar" probability $P_{\mu e}^{S}$. However, since $P_{\mu e}^{S}$ itself is very small, the correction to the total probability via $P_{\mu e}^{S}$ can be neglected.

The correction due to $r_{V}-1$ is given by

$$
\frac{\delta P_{\mu e}^{I}}{P_{\mu e}^{I}}=r_{V}-1 .
$$


Since $P_{\mu e}^{I} \approx(0.25-0.30) P_{\mu e}$, the correction can be estimated as $(0.25-0.30)\left(r_{V}-1\right)$. According to the computations in section 4.2, this can lead up to (10-15)\% correction for $\epsilon_{e \mu}$ and $(15-22) \%$ for $\epsilon_{e \tau}$ at T2K in the $1 \sigma$ allowed intervals. In comparison, the corrections are about 12 times smaller at $\mu$ SK. They strongly depend on the phases of $\epsilon_{\alpha \beta}$ and the phase $\delta_{D}$.

The correction due to $\delta_{2}$ is given by

$$
\frac{\delta P_{\mu e}^{I}}{P_{\mu e}^{I}}=\tan \left(\delta_{D}-\delta_{2}-\phi_{32}^{m}\right) \delta_{2}
$$

with $\delta_{2}$ defined in (4.14) being roughly proportional $\mathbb{I}\left(\epsilon_{12}^{\prime}\right)$. Then the correction to the total probability is suppressed by a factor of $\tan \left(\delta_{D}-\delta_{2}-\phi_{32}^{m}\right) \approx 1 / 4 \sim 1 / 3$. As a result, the contribution from $\delta_{2}$ is somewhat smaller than that from $\left(r_{V}-1\right)$, reaching $(5-10) \%$ at $\mathrm{T} 2 \mathrm{~K}$.

2. The correction due to the NSI matter effect on the 1-3 mixing. Using eqs. (4.30) and (4.10), we can write the correction to the mixing angle in matter $\theta_{13}^{m, S I}$ with standard interaction as

$$
\delta \theta_{13}^{m}=\theta_{13}^{m}-\theta_{13}^{m, S I}=x_{31}\left[\cos \delta_{3}\left|s_{13} c_{13}+\epsilon_{13}^{\prime}\right|-s_{13} c_{13}\right] .
$$

Thus, $\delta \theta_{13}^{m}$ depends on $\left(\epsilon_{12}^{\prime}, \epsilon_{13}^{\prime}\right) \sim \lambda$ (i.e., on different combination of the NSI parameters). According to (4.46), $\delta \theta_{13}^{m} \sim B s_{13} r_{\Delta}$ with $B \sim 4-6$ at T2K and $B \approx 1$ at $\mu$ SK. The corrections to the 1-3 mixing can contribute to the "atmospheric" probability as

$$
\frac{\delta P_{\mu e}^{A}}{P_{\mu e}^{A}} \approx 4 \cot 2 \theta_{13} \delta \theta_{13}^{m} \sim B r_{\Delta} .
$$

For the interference term the relative correction is 2 times smaller and the contribution to the total probability is further suppressed by a factor $1 / 4 \sim 1 / 3$. The total correction to $P_{\mu e}$ due to $\delta \theta_{13}^{m}$ can be as large as $(10 \sim 15) \%$.

3. The correction via the 1-3 oscillation phase. The modification of the $3-1$ phase due to NSI is

$$
\delta \phi_{13}^{m}=\phi_{13}^{m, N S I}-\phi_{13}^{m}=\left(\Delta H_{31}^{N S I}-\Delta H_{31}\right) \frac{L}{2} .
$$

Using (4.15) we obtain

$$
\Delta H_{31}-\Delta H_{31}^{0}=\frac{1}{2} V\left(2 \epsilon_{33}^{\prime}-\epsilon_{11}^{\prime}-\epsilon_{22}^{\prime}\right)+\frac{1}{2}\left[\Delta H_{21}-\Delta H_{21}^{0}\right],
$$

where $\Delta H_{21}$ is defined in (4.14), $\Delta H_{31}^{0}, \Delta H_{21}^{0}$ are splittings between the eigenvalues without NSI $(\epsilon=0)$. In turn, being in general of the order $V \epsilon$, see (4.14), $\Delta H_{21}-\Delta H_{21}^{0} \approx-V \cos 2 \theta_{12} \epsilon_{11}^{\prime}$ below the 1-2 resonance and $\Delta H_{21}-\Delta H_{21}^{0} \rightarrow V \epsilon_{11}^{\prime}$ above the resonance. In the second case there is a cancellation between the last term in (4.49) with $V \epsilon_{11}^{\prime}$ in the first term there. Thus, the phase difference becomes

$$
\delta \phi_{13}^{m} \approx h \frac{V L}{4} \sim r_{\Delta},
$$


where the coefficient $h$ is smaller than 1 and can be suppressed at high energies. Notice that $\delta \phi_{13}^{m}$ depends not only on $\epsilon_{11}^{\prime}=O(1)$ but also on other diagonal elements $\epsilon_{22}^{\prime}, \epsilon_{11}^{\prime} \sim \lambda^{2}$. So, $\delta \phi_{13}^{m} \sim r_{\Delta}$.

The correction to the "atmospheric" probability equals

$$
\frac{\delta P_{\mu e}^{A}}{P_{\mu e}^{A}} \approx 2 \cot \phi_{13} \delta \phi_{13}^{m} .
$$

At the first oscillation maximum an additional suppression comes from $\cot \phi_{13} \approx 0$.

The analytic consideration presented here allows us to reproduce the results of numerical computations and analyse their dependence on the NSI parameters. In general, the violation of vacuum mimicking gives substantial or even dominant contribution to the probability change. The largest corrections come from $r_{V}-1, \delta_{2}$, and $\delta \theta_{13}^{m}$. Their relative contributions depend on the phases of $\epsilon$ 's. For $\operatorname{Arg}\left[\epsilon_{e \mu}\right] \sim 0$ or $\pi$ the violation of vacuum mimicking gives the main contribution, which is however suppressed at $\operatorname{Arg}\left[\epsilon_{e \mu}\right] \sim \pi / 2,3 \pi / 2$. If the NSI parameters are such that $\epsilon_{12}^{\prime}=0$, the main contribution to $P_{\mu e}$ comes from the 1-3 mixing determined by $\epsilon_{13}^{\prime}$. And vice versa if $\epsilon_{13}^{\prime}=0$, the effect of 1-3 mixing is suppressed and contributions from $r_{V}-1$ and $\delta_{2}$ dominate, etc.

These features are in agreement with our numerical computations. With all the contributions combined, the total effect of NSI parameters is shown in figure 5. Although the NSI parameter $\epsilon_{e e}-\epsilon_{\mu \mu}$ does not contribute to the deviation from vacuum mimicking (see figure 3) or to the leptonic Dirac CP phase (see figure 4), it affects the total oscillation probability $P_{\mu e}$, mainly due to the correction $\delta \theta_{13}^{m}$ in (4.47), which is suppressed by $s_{13}$. The main contribution to the corrections comes from $\epsilon_{e \mu}$ and $\epsilon_{e \tau}$, reaching $20 \%$ and $30 \%$, respectively, for the $1 \sigma$ upper bounds. The contribution from $\left(\epsilon_{e e}-\epsilon_{\mu \mu}, \epsilon_{\tau \tau}-\epsilon_{\mu \mu}, \epsilon_{\mu \tau}\right)$ can be as large as $(5-10) \%$.

\section{CP measurement in the presence of non-standard interactions}

In this section we use T2K and $\mu \mathrm{SK}$ as examples to illustrate the NSI effect on the measurement of the genuine Dirac CP phase $\delta_{D}$. To estimate the CP sensitivity we have simulated the pseudo-data at T2K and $\mu \mathrm{DAR}$ for presently favored "true" value $\delta_{D}=3 \pi / 2$. We take the following values of the neutrino oscillation parameters

$$
\begin{aligned}
& \sin ^{2} 2 \theta_{13}=0.089 \pm 0.005, \quad \sin ^{2} 2 \theta_{12}=0.857 \pm 0.024, \quad \sin ^{2} 2 \theta_{23}=0.5 \pm 0.055, \\
& \Delta m_{21}^{2}=(7.5 \pm 0.2) \times 10^{-5} \mathrm{eV}^{2}, \quad \Delta m_{31}^{2}=(2.4 \pm 0.1) \times 10^{-3} \mathrm{eV}^{2},
\end{aligned}
$$

to be consistent with the setup in [44]. The pseudo-data is simulated in the absence of NSI.

We use the following $\chi^{2}$ function

$$
\chi^{2} \equiv \chi_{\text {stat }}^{2}+\chi_{\text {sys }}^{2}+\chi_{\text {prior }}^{2}
$$

to fit the pseudo-data, with or without NSI. Here $\chi_{\text {stat }}^{2}$ corresponds to statistical errors: $\chi_{\text {stat }}^{2} \equiv \sum_{i}\left(N_{i}^{\text {data }}-N_{i}^{\text {fit }}\right)^{2} / N_{i}^{\text {data }}$ while $\chi_{\text {sys }}^{2}=\sum_{i}\left[\left(f_{i}-1\right) / \delta f\right]^{2}$ accounts for the systematical 

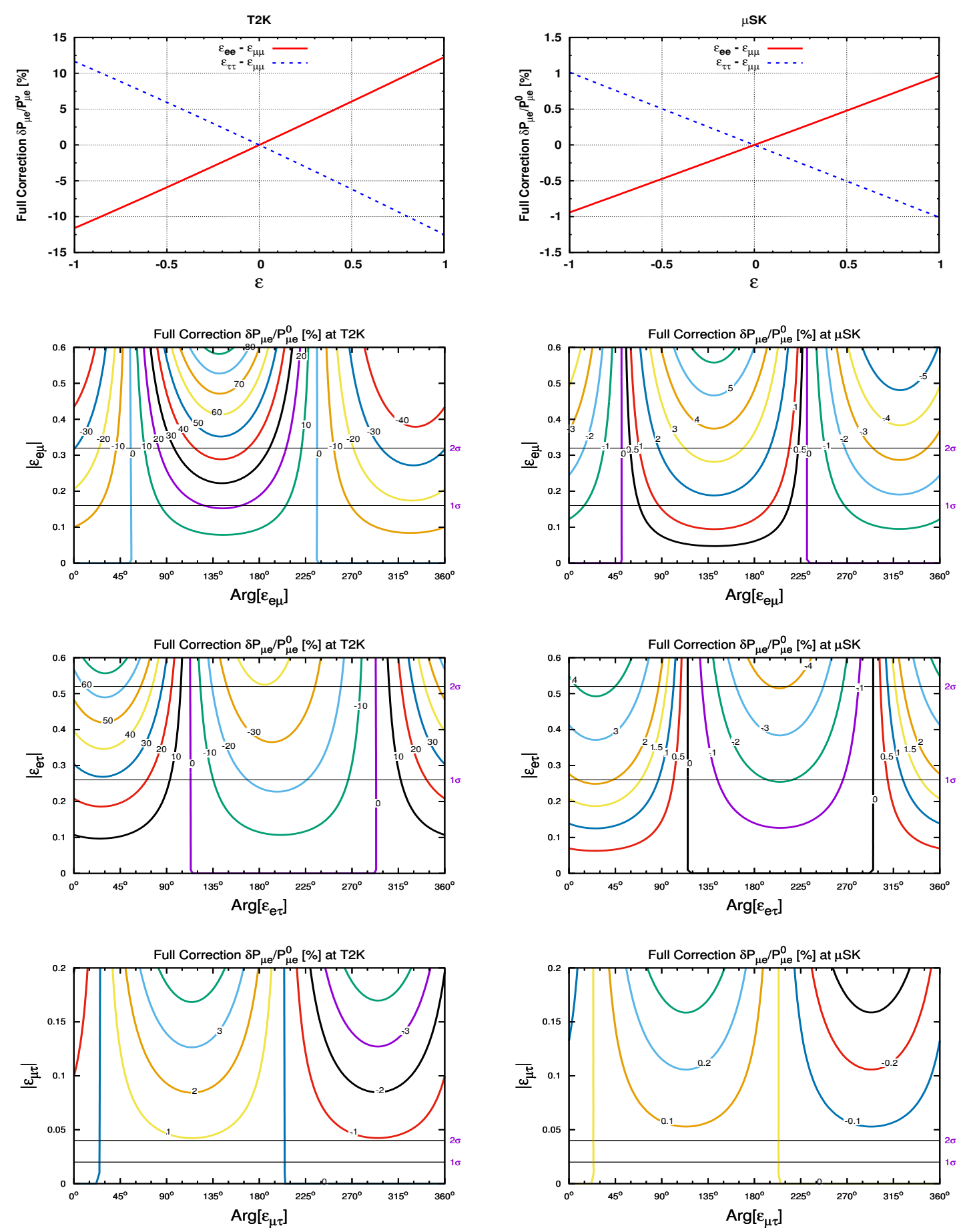

Figure 5. The dependence of the corrections to total oscillation probability $P_{\mu e}$ on values of NSI parameters: (a) $\epsilon_{e e}-\epsilon_{\mu \mu}$ and $\epsilon_{\tau \tau}-\epsilon_{\mu \mu} ;$ (b) $\epsilon_{e \mu}$; (c) $\epsilon_{e \tau} ;$ (d) $\epsilon_{\mu \tau}$ with $\delta_{D}=-90^{\circ}$ and $\rho_{m}=3 \mathrm{~g} / \mathrm{cm}^{3}$. The left panels are for $E_{\nu}=600 \mathrm{MeV}$ and $L=295 \mathrm{~km}$ at $\mathrm{T} 2(\mathrm{H}) \mathrm{K}$ while the right panels are for $E_{\nu}=50 \mathrm{MeV}$ and $L=23 \mathrm{~km}$ at $\mu \mathrm{SK}$. 
uncertainty in the neutrino fluxes from the J-PARC and $\mu$ DAR neutrino sources. The JPARC flux has $5 \%$ uncertainty for both neutrino and anti-neutrino modes, also for the $\mu$ DAR flux we take about $5 \%^{2}$ uncertainty, see [44] for details. Finally, the prior part $\chi_{\text {prior }}^{2}$ takes into account our current knowledge of the neutrino oscillation parameters in vacuum (5.1) and the prior constraints (4.2) on the NSI parameters. The later is included only when we fit the pseudo-data with the NSI parameters.

In simulations we used both the neutrino and antineutrino channels. Recall that the standard oscillation probability (2.33) at the first oscillation maximum corresponding to $\phi_{31}^{m}=\pi / 2$ and $\phi_{21}^{m} \approx r_{\Delta} \times \pi / 2$ can be approximated as

$$
P_{\mu e} \approx s_{23}^{2} \sin ^{2} 2 \theta_{13}^{m} \pm c_{13}^{m} \sin 2 \theta_{13}^{m} \sin 2 \theta_{23} \sin 2 \theta_{12} \frac{\pi}{2} r_{\Delta} \sin \delta_{D}
$$

where \pm stand for neutrinos and anti-neutrinos, respectively. The first term in (5.3) is larger than the second term by a factor of $s_{13} / r_{\Delta} \sim 5$. This magnifies the effect of the uncertainties in the first term. The largest one appears in the 2-3 mixing, $s_{23}$. Around the maximal mixing, $\sin 2 \theta_{23} \approx 1$, the variations of $s_{23}^{2}$ are enhanced with respect to the variations of $\sin 2 \theta_{23}: \Delta\left(s_{23}^{2}\right) \approx \Delta\left(\sin 2 \theta_{23}\right) \times\left(\sin 2 \theta_{23} / 2 \cos 2 \theta_{23}\right) \gg \Delta\left(\sin 2 \theta_{23}\right)$. Consequently, a small uncertainty in $\sin 2 \theta_{23}$ propagates to a large uncertainty in $s_{23}$. It effectively reduces the sensitivity to $\delta_{D}$ which originates from the second term in (5.3). Running T2K in both neutrino and anti-neutrino modes can avoid this problem. With both $P_{\mu e}$ and $P_{\bar{\mu} \bar{e}}$ measured, the difference between them is purely the CP violating term that is proportional to $\sin \delta_{D}$.

The results in this section are simulated by the NuPro package [65].

\subsection{The CP sensitivity at T2K in the presence of NSI}

In simulations for $\mathrm{T} 2 \mathrm{~K}$ we use $7.8 \times 10^{21}$ proton on target (POT) for the J-PARC flux, corresponding to 342 events in the neutrino mode or 83 events in the anti-neutrino mode for $\delta_{D}=3 \pi / 2$ [66]. As discussed above, to reduce uncertainties in the determination of $\delta_{D}$ at $\mathrm{T} 2 \mathrm{~K}$, it is necessary to run the experiment in both neutrino and anti-neutrino modes. However, in both production and detection, the cross sections for anti-neutrinos are smaller than those for neutrinos. To collect comparable number of events, and hence balance the statistical uncertainty in both modes, the antineutrino mode should run longer. Therefore, in simulations we use 2 years of running in the neutrino mode and 4 years in the antineutrino mode. Correspondingly, 114 neutrino events and 56 antineutrino events are expected.

We compute the minimal $\chi^{2}$ value as a function of the fit value of the phase $\delta_{D}^{\mathrm{fit}}$, with and without the NSI parameters. The results are shown in figure 6 . The solid black line in figure 6 presents the CP sensitivity at T2K without NSI. We can see that this line is nearly symmetric with respect to $\delta_{D}^{\text {fit }}=\delta_{D}^{\text {true }}=3 \pi / 2$ which is related to the degeneracy between $\delta_{D}$ and $\pi-\delta_{D}$ due to the $\sin \delta_{D}$ dependence in the oscillation probability (5.3). According to figure 6 , the true value $\delta_{D}^{\text {true }}=3 \pi / 2$ can be distinguished from $\delta_{D}^{\text {fit }}=0(\pi)$ by $\chi^{2}=3(1.7 \sigma)$ and from $\delta_{D}^{\text {fit }}=\pi / 2$ by $\chi^{2}=15(3.8 \sigma)$. The results can be easily scaled for higher statistics at T2HK or T2K-II.

\footnotetext{
${ }^{2}$ If the near detector $\mu$ Near [64] is also implemented, the $\mu$ DAR flux uncertainty can be significantly reduced.
} 


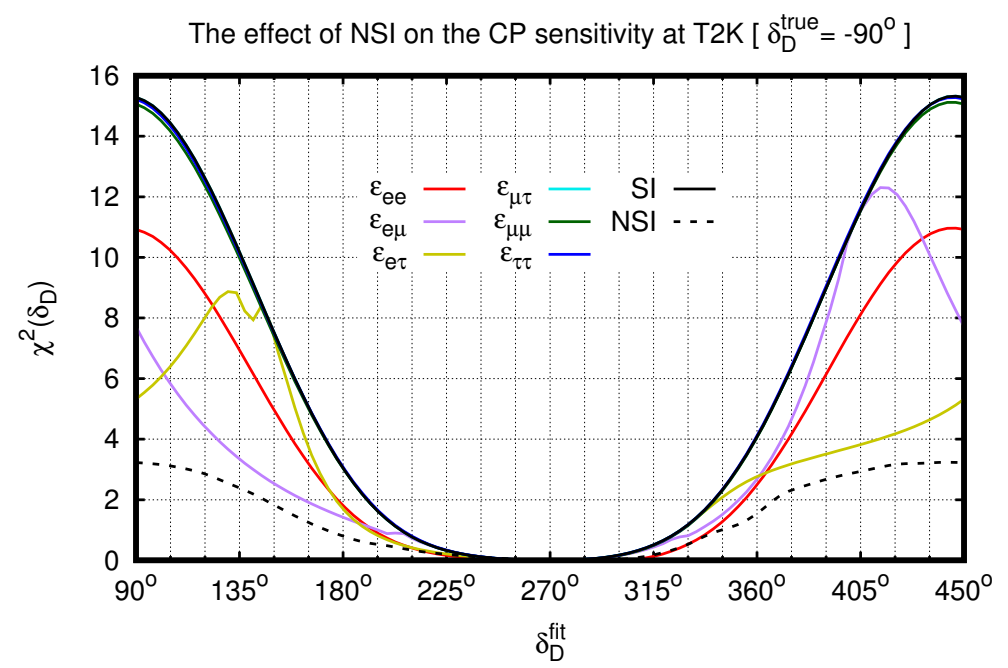

Figure 6. The effect of NSI on the CP sensitivity at T2K with 2 years of running for the neutrino mode and 4 years for the antineutrino mode, with the prior constraints (4.2). The pseudo-data with standard interaction and $\delta_{D}^{\text {true }}=3 \pi / 2$ is fit with standard interaction (SI) as well as with individual NSI parameters $\left(\epsilon_{e e}, \epsilon_{e \mu}, \epsilon_{e \tau}, \epsilon_{\mu \tau}, \epsilon_{\mu \mu}, \epsilon_{\tau \tau}\right)$ and full set of NSI parameters (dashed line).

The sensitivity to $\delta_{D}$ can be significantly undermined by NSI. In figure 6 we show the dependence of $\chi^{2}$ on the fit value $\delta_{D}^{\text {fit }}$ for different NSI parameters separately, although only two combinations of the three diagonal elements $\left(\epsilon_{e e}, \epsilon_{\mu \mu}\right.$, and $\left.\epsilon_{\tau \tau}\right)$ affect oscillations, as we used in section 2. The color curves in figure 6 show that the CP sensitivity at T2K is significantly reduced by $\epsilon_{e e}, \epsilon_{e \mu}$, and $\epsilon_{e \tau}$, but almost not affected by $\epsilon_{\mu \mu}, \epsilon_{\mu \tau}, \epsilon_{\tau \tau}$. This is because $\epsilon_{\tau \tau}-\epsilon_{\mu \mu}$ and $\epsilon_{\mu \tau}$ are constrained much stronger according to (4.2) and also their contributions to $\epsilon_{12}^{\prime}$ are suppressed by $s_{13}$, see (4.6). Consequently, they have small contribution to the violation of vacuum mimicking via $r_{V}(4.32)$ and to the Dirac CP phase via $\delta_{2}(4.14)$. Although $\epsilon_{e e}-\epsilon_{\mu \mu}$ cannot contribute to the leading order corrections via the Dirac CP phase $\delta_{D}^{m}$ and $r_{V}$, see (4.39), its effect on the oscillation probability via $\delta \theta_{13}^{m}(4.46)$ as a function of $\epsilon_{13}^{\prime}$ (4.6) can still be significant due to weak prior constraint (4.2). For $\epsilon_{e \mu}$ and $\epsilon_{e \tau}$, the prior constraints are stronger but their contributions are not suppressed in $\epsilon_{12}^{\prime}$, see (4.6).

The non-vanishing $\epsilon_{e e}, \epsilon_{e \mu}, \epsilon_{e \tau}$ reduce the distinguishability between $\delta_{D}^{\text {fit }}=0(\pi)$ and $\delta_{D}^{\text {true }}=3 \pi / 2$ from $\chi^{2}=3$ to $1.7(1.3 \sigma)$. The difference between the two maximal CP values $\delta_{D}= \pm \pi / 2$ decreases from $\chi^{2} \approx 15$ (roughly $4 \sigma$ ) to $\chi^{2} \approx 5.5(2.3 \sigma)$. If all NSI parameters are present, the $\mathrm{CP}$ sensitivity at $\mathrm{T} 2 \mathrm{~K}$ can be further reduced as is shown by the dashed black line in figure 6 . The distinguishability of $\delta_{D}^{\text {fit }}=\pi / 2$ from $\delta_{D}^{\text {true }}=3 \pi / 2$ is reduced by roughly a factor of 5 to $\chi^{2} \approx 3.2(1.8 \sigma)$ and for $\delta_{D}^{\text {fit }}=0(\pi)$ it becomes $\chi^{2} \approx 0.8(0.9 \sigma)$. The value of $\chi^{2}$ for $\delta_{D}^{\mathrm{fit}}=0(\pi)$ and the corresponding best-fit values of the NSI parameters are shown in table 1.

\subsection{Improving the CP sensitivity with neutrinos from $\mu \mathrm{DAR}$}

Due to smaller energies, $\sim 50 \mathrm{MeV}$, as compared with the peak energies, $\sim 600 \mathrm{MeV}$, of the J-PARC flux, neutrinos of the $\mu$ DAR flux will experience 12 times smaller matter effect 


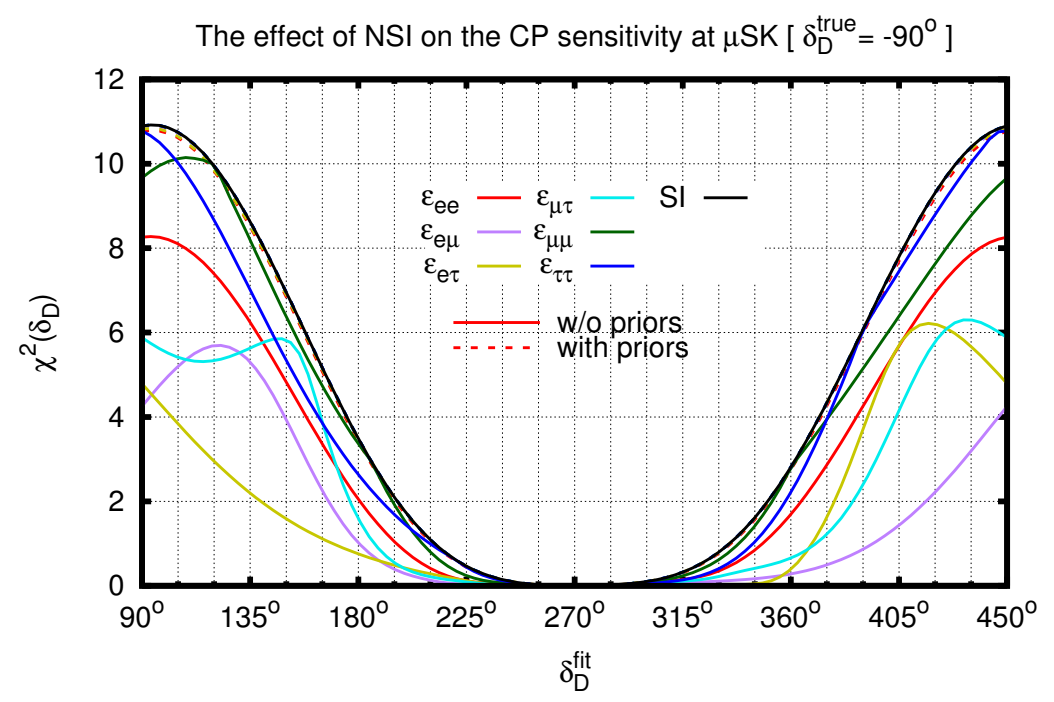

Figure 7. The effect of NSI on the CP sensitivity at $\mu$ SK with 6 years of running in the antineutrino mode, without (solid lines) and with (dashed line) the prior constraints (4.2).

than in $\mathrm{T} 2(\mathrm{H}) \mathrm{K}$. In simulation we use the characteristics proposed for $\mu \mathrm{SK}$ [44] which includes the existing Super-K detector and a $9 \mathrm{~mA}$ cyclotron to produce $\bar{\nu}_{\mu}$ from $\mu \mathrm{DAR}$. The optimal distance between the $\mu \mathrm{DAR}$ source and the SK detector is around $23 \mathrm{~km}$. For 6 years of running, a total number $1.1 \times 10^{25}$ POT can be collected, corresponding to 212 $\bar{\nu}_{\mu} \rightarrow \bar{\nu}_{e}$ oscillation events in the SK detector for $\delta_{D}^{\text {true }}=3 \pi / 2$.

In figure 7 , we show the CP sensitivity at $\mu \mathrm{SK}$. Without NSI the true value $\delta_{D}^{\text {true }}=3 \pi / 2$ can be distinguished from $\delta_{D}^{\text {fit }}=0$ or $\pi$ by $\chi^{2}=2.8(1.7 \sigma)$ and from $\delta_{D}^{\text {fit }}=\pi / 2$ by $\chi^{2}=11$ $(3.3 \sigma)$. Notice that the curve is not symmetric with respect to $\delta_{D}=3 \pi / 2$ due to the interplay between the $\sin \delta_{D}$ and $\cos \delta_{D}$ dependences in the oscillation probability $P_{\bar{\mu} \bar{e}}$.

The matter effects at $\mu \mathrm{SK}$ are an order of magnitude smaller than at T2K, and consequently the sensitivity to $\delta_{D}$ is less affected by NSI, in comparison with figure 6 . With the prior constraint (4.2) imposed, the sensitivity to $\delta_{D}$ at $\mu \mathrm{SK}$ is almost unaffected. The sensitivity to $\delta_{D}$ is significantly reduced by the NSI parameters only if no prior constraint is imposed. Even in this case the sensitivity to $\delta_{D}$ is not reduced to almost zero by the NSI parameters $\left(\epsilon_{e e}, \epsilon_{e \mu}, \epsilon_{e \tau}\right)$, which is the case at T2K.

Let us consider the sensitivity to $\delta_{D}$ of the combined measurements at TNT2K [44]. While T2K unavoidably measures both $\delta_{D}$ and NSI, $\mu$ SK mainly provides a determination of the phase $\delta_{D}$. The figure 8 shows how $\mu \mathrm{SK}$ can improve the sensitivity to $\delta_{D}$, in comparison with figure 6 from T2K only. With the prior constraints (4.2) imposed, the significance of distinguishing $\delta_{D}^{\text {true }}=3 \pi / 2$ from $\delta_{D}^{\text {fit }}=\pi / 2$ can reach $5 \sigma$ and the distinguishability from vanishing $\mathrm{CP}$ values, $\delta_{D}=0$ or $\pi$, can be about $2.5 \sigma$ for individual NSI parameters. The numbers become $4.6 \sigma$ and $2.2 \sigma$ with the full set of NSI parameters, in comparison with $1.8 \sigma$ and $0.9 \sigma$ at $\mathrm{T} 2 \mathrm{~K}$ as well as $3.3 \sigma$ and $1.7 \sigma$ at $\mu \mathrm{SK}$. Combining the T2K and $\mu \mathrm{SK}$ results will substantially improve the sensitivity to $\delta_{D}$, in comparison to the sensitivity of $\mathrm{T} 2 \mathrm{~K}$ or $\mu \mathrm{SK}$ alone. 

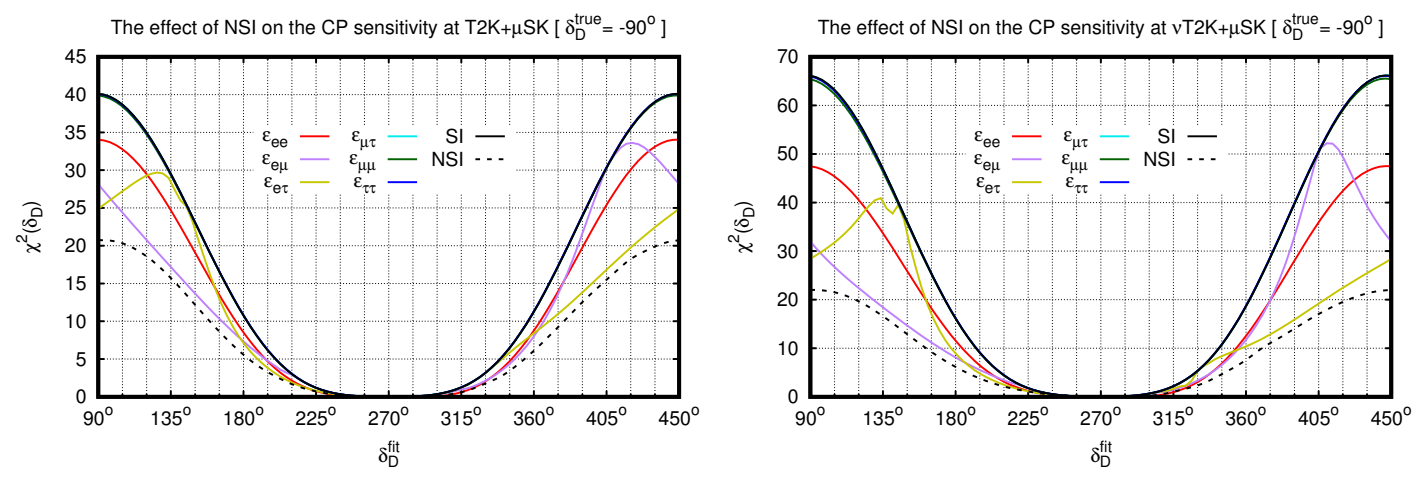

Figure 8. The effect of NSI on the CP sensitivity at TNT2K with the prior constraints (4.2). For comparison, the same configuration of $\mathrm{T} 2 \mathrm{~K}$ as in figure 6,2 years of running in the neutrino mode and 4 years for anti-neutrino, has adopted in the left panel (T2K) while the right takes 6 years of running in the neutrino mode $(\nu \mathrm{T} 2 \mathrm{~K})$. For both panels, the $\mu \mathrm{SK}$ experiment runs for 6 years in the anti-neutrino mode. The pseudo-data with standard interaction and $\delta_{D}^{\text {true }}=-90^{\circ}$ is fit with standard interaction (SI), individual NSI parameters $\left(\epsilon_{e e}, \epsilon_{e \mu}, \epsilon_{e \tau}, \epsilon_{\mu \tau}, \epsilon_{\mu \mu}, \epsilon_{\tau \tau}\right)$, or full set of NSI parameters (dashed line).

With a $\mu$ DAR antineutrino source built near the Super-K/Hyper-K detectors, T2(H)K can devote all its exposure time to the neutrino run. This can significantly increase the event number. We can obtain a factor of 3 increase in the number of neutrino events and a factor of 4 increase in the number of antineutrino events in comparison to those at T2K. In addition, the wide energy spectrum of the $\mu$ DAR antineutrino will break the degeneracy between $\delta_{D}$ and $\pi-\delta_{D}$ by providing $\cos \delta_{D}$ dependence of $P_{\mu e}$, especially for non-maximal CP phase, $\delta_{D} \neq \pi-\delta_{D}$. If the CP phase happens to be $\delta_{D}= \pm \pi / 2, \mu$ SK can also reduce the uncertainty around $\delta_{D}^{\text {true }}=3 \pi / 2$ [44]. With mainly $\sin \delta_{D}$ dependence at T2K, the CP uncertainty $\Delta\left(\delta_{D}\right) \propto 1 / \cos \delta_{D}$ diverges for maximal CP violation. This can be significantly improved due to the $\cos \delta_{D}$ dependence provided by the $\mu \mathrm{DAR}$ flux.

In the right panel of figure 8 we show the $\mathrm{CP}$ sensitivity of the combined measurement with T2K running 6 years purely in the neutrino mode $(\nu \mathrm{T} 2 \mathrm{~K})$ and $\mu$ SK running 6 year purely in the antineutrino mode. The latter $\mathrm{CP}$ sensitivity is higher than the sensitivity of the combination of T2K (neutrino and antineutrino) and $\mu \mathrm{SK}$. Without NSI, the distinguishability of $\delta_{D}^{\text {fit }}=\pi / 2$ from $\delta_{D}^{\text {true }}=3 \pi / 2$ increases from around $6 \sigma$ to around $8 \sigma$. After including individual NSI parameter, it increases from $5 \sigma$ to $5.4 \sigma$ with prior constraints. The distinguishability of $\delta_{D}^{\text {fit }}=0$ or $\pi$ from $\delta_{D}^{\text {true }}=3 \pi / 2$ increases from $3.3 \sigma$ to $4 \sigma$ for SI and from $2.6 \sigma$ to $2.8 \sigma$ for individual NSI parameter with prior constraints. Thus the combination of $\nu \mathrm{T} 2 \mathrm{~K}$ (fully neutrino running) and $\mu \mathrm{SK}$, is better than splitting the $\mathrm{T} 2 \mathrm{~K}$ run among neutrino and antineutrino modes. Comparing the solid black curve for SI and the dashed one for the full set of NSI parameters, we find that the distinguishability of $\delta_{D}^{\text {fit }}=\pi / 2$ from $\delta_{D}^{\text {true }}=3 \pi / 2$ decreases from $\chi^{2} \approx 40$ to 21 at T2K $(2+4$ years $)+\mu \mathrm{SK}$, while it decreases from around 65 to 22 for $\nu \mathrm{T} 2 \mathrm{~K}+\mu \mathrm{SK}$. In both cases the CP violation can be established at more than $4.5 \sigma$. Although the CP sensitivity with NSI is roughly the same for $\mathrm{T} 2 \mathrm{~K}+\mu \mathrm{SK}$ and $\nu \mathrm{T} 2 \mathrm{~K}+\mu \mathrm{SK}$, it can be significantly increased at $\nu \mathrm{T} 2 \mathrm{~K}$ if there is no NSI. 


\begin{tabular}{|c|cc|cc|cc|cc|}
\hline $\begin{array}{c}\delta_{D}^{\text {true }}=-90^{\circ} \text { vs } \delta_{D}^{\mathrm{fit}}=0^{\circ} \\
\text { Event Numbers }\end{array}$ & \multicolumn{2}{|c|}{$\mathrm{T} 2 \mathrm{~K}$} & \multicolumn{2}{|c|}{$\mu \mathrm{SK}$} & \multicolumn{2}{c|}{$\mathrm{T} 2 \mathrm{~K}+\mu \mathrm{SK}$} & \multicolumn{2}{c|}{$\nu \mathrm{T} 2 \mathrm{~K}+\mu \mathrm{SK}$} \\
\hline$\chi^{2}$ for SI \& NSI & 4.08 & 1.54 & 2.81 & 2.75 & 11.3 & 6.10 & 18.7 & 7.59 \\
\hline$\chi^{2}$ & 2.50 & - & 2.75 & - & 8.77 & - & 12.5 & - \\
$\epsilon_{e e}^{\mathrm{bf}}$ & 0.69 & 0.57 & 0.48 & 0.47 & 0.81 & 0.63 & 1.07 & 0.70 \\
\hline$\chi^{2}$ & 4.06 & - & 2.81 & - & 11.3 & - & 18.6 & - \\
$\epsilon_{\mu \mu}^{\mathrm{bf}}$ & -0.02 & -0.01 & -0.02 & -0.01 & -0.02 & -0.01 & -0.02 & -0.01 \\
\hline$\chi^{2}$ & 4.11 & - & 2.81 & - & 11.3 & - & 18.7 & - \\
$\epsilon_{\tau \tau}^{\mathrm{bf}}$ & 0.02 & 0.01 & 0.02 & 0.01 & 0.02 & 0.01 & 0.01 & 0.01 \\
\hline$\chi^{2}$ & 2.68 & - & 2.81 & - & 8.01 & - & 11.7 & - \\
$\epsilon_{e \mu}^{\mathrm{bf}}$ & 0.12 & 0.07 & 0.01 & 0.01 & 0.19 & 0.11 & 0.23 & 0.10 \\
\hline$\chi^{2}$ & 2.77 & - & 2.81 & - & 8.42 & - & 10.4 & - \\
$\epsilon_{e \tau}^{\mathrm{bf}}$ & 0.25 & 0.14 & 0.01 & 0.01 & 0.37 & 0.21 & 0.51 & 0.30 \\
\hline$\chi^{2}$ & 4.08 & - & 2.81 & - & 11.3 & - & 18.7 & - \\
$\epsilon_{\mu \tau}^{\mathrm{bf}}$ & 0 & 0 & 0 & 0 & 0 & 0 & 0 & 0 \\
\hline
\end{tabular}

Table 1. The $\chi_{\min }^{2}$ and best fit values of the NSI parameters when using $\delta_{D}^{\mathrm{fit}}=0^{\circ}$ to fit the pseudodata generated with SI and $\delta_{D}^{\text {true }}=-90^{\circ}$, under the prior constraints (4.2). In each experimental configuration $\mathrm{T} 2 \mathrm{~K}, \mu \mathrm{SK}, \mathrm{T} 2 \mathrm{~K}+\mu \mathrm{SK}$, and $\nu \mathrm{T} 2 \mathrm{~K}+\mu \mathrm{SK}$, the $\chi^{2}$ for SI comes from the fit with standard interaction, NSI with either individual or the full set of $\left(\epsilon_{e e}, \epsilon_{e \mu}, \epsilon_{e \tau}, \epsilon_{\mu \tau}, \epsilon_{\mu \mu}, \epsilon_{\tau \tau}\right)$.

\section{Conclusions}

We explored the CP violation and the matter effects in neutrino oscillation in the presence of standard and non-standard interactions at low energies and relatively small baselines. This experimental setup is realized in $\mathrm{T} 2 \mathrm{~K}$, the experiments based on $\mu \mathrm{DAR}$, etc. A simple analytic formalism is elaborated which describes the NSI effects and their dependence on the parameters of NSI interactions. The vacuum mimicking and its violation in the 1-2 sector as well as the use of the separation basis play central roles in the formalism.

In the case of standard interactions due to vacuum mimicking the matter affects the oscillation probability $P_{\mu e}$ mainly via the correction to the 1-3 mixing. We find that matter changes the probability by about $13 \%$ at T2K and $1 \%$ at $\mu$ DAR. Also we show that vacuum mimicking provides a simple explanation of the fact that the usual formula for $3 \nu-$ oscillation probability gives a very good description at energies around the 1-2 resonance.

In the presence of NSI, the vacuum mimicking in the 1-2 sector is strongly broken. The breaking shows up in a very specific way and is related to the single NSI parameter $\epsilon_{12}^{\prime}$ in the separation basis. The same parameter $\epsilon_{12}^{\prime}$ controls the additional contribution to the effective CP phase in matter. The 1-3 mixing is modified by another parameter $\epsilon_{13}^{\prime}$ while the 1-3 oscillation phase is corrected by the diagonal elements $\epsilon_{11}^{\prime}, \epsilon_{22}^{\prime}$ and $\epsilon_{33}^{\prime}$.

We show that the total probability $P_{\mu e}$ is mainly affected by the violation of vacuum mimicking parametrized by $\left(r_{V}-1\right)$ and $\delta_{2}$ as well as by the correction to the 1-3 mixing, $\delta \theta_{13}^{m}$. The relative effects of the violation of vacuum mimicking and $\delta \theta_{13}^{m}$ on $P_{\mu e}$ depend 
on the phases of $\epsilon_{e \mu}^{\prime}$ or/and $\epsilon_{e \tau}^{\prime}$. Within the $1 \sigma$ intervals, the correction to $P_{\mu e}$ due to NSI can reach $(20-30) \%$ at $\mathrm{T} 2 \mathrm{~K}$ and $2 \%$ at $\mu \mathrm{DAR}$. The corrections to the $\mathrm{CP}$ phase can be as large as $(40-50)^{\circ}$ at $\mathrm{T} 2 \mathrm{~K}$ and $3^{\circ}$ at $\mu \mathrm{DAR}$.

We apply our analytic formalism to the CP phase measurement at low energies. The standard interaction leads to vacuum mimicking in the solar amplitude and keeps the Dirac CP phase $\delta_{D}$ unaffected. On the contrary, NSI can introduce significant deviation from vacuum mimicking, and consequently, modify $\delta_{D}$ to practically any value at T2K. With prior constraints (4.2) on the size of NSI parameters, the CP sensitivity in terms of $\chi^{2}\left(\delta_{D}\right)$ at T2K can be reduced by almost a factor of $2 \sim 3$. The effect of NSI can be even larger at $\mathrm{NO} \nu \mathrm{A}$ and DUNE with higher neutrino energies.

We show that TNT2K - the combination of T2K and the new component $\mu \mathrm{SK}$ can resolve the $\delta_{D}$-NSI degeneracy. The $\mu$ SK component uses the SK detector to study the oscillations of the antineutrinos from a $\mu \mathrm{DAR}$ source. Since the $\mu \mathrm{DAR}$ flux has 10 times lower energy, the effect of NSI at $\mu \mathrm{SK}$ is much smaller than at T2K. While T2K measures both the genuine CP phase $\delta_{D}$ and NSI, $\mu \mathrm{SK}$ can provide clean determination of $\delta_{D}$ simultaneously. The sensitivity to $\delta_{D}$ which can be achieved by this combination of $\mathrm{T} 2 \mathrm{~K}$ and $\mu \mathrm{SK}$ is much higher than the sensitivity of T2K or $\mu \mathrm{SK}$ alone. The TNT2K configuration can guarantee high sensitivity to $\delta_{D}$ in the presence of NSI.

\section{Acknowledgments}

SFG would like to thank Manfred Lindner for various discussions as well as Hong-Jian He and Center for High Energy Physics at Tsinghua University for hospitality during the preparation of this paper. A.S. is grateful to E. K. Akhmedov for useful discussions.

Open Access. This article is distributed under the terms of the Creative Commons Attribution License (CC-BY 4.0), which permits any use, distribution and reproduction in any medium, provided the original author(s) and source are credited.

\section{References}

[1] L. Wolfenstein, Neutrino oscillations in matter, Phys. Rev. D 17 (1978) 2369 [InSPIRE].

[2] M.M. Guzzo, A. Masiero and S.T. Petcov, On the MSW effect with massless neutrinos and no mixing in the vacuum, Phys. Lett. B 260 (1991) 154 [INSPIRE].

[3] E. Roulet, MSW effect with flavor changing neutrino interactions, Phys. Rev. D 44 (1991) R935 [INSPIRE].

[4] T. Ohlsson, Status of non-standard neutrino interactions, Rept. Prog. Phys. 76 (2013) 044201 [arXiv: 1209.2710] [INSPIRE].

[5] O.G. Miranda and H. Nunokawa, Non standard neutrino interactions: current status and future prospects, New J. Phys. 17 (2015) 095002 [arXiv:1505.06254] [INSPIRE].

[6] M.C. Gonzalez-Garcia, Y. Grossman, A. Gusso and Y. Nir, New CP-violation in neutrino oscillations, Phys. Rev. D 64 (2001) 096006 [hep-ph/0105159] [INSPIRE]. 
[7] A.M. Gago, H. Minakata, H. Nunokawa, S. Uchinami and R. Zukanovich Funchal, Resolving $C P$-violation by standard and nonstandard interactions and parameter degeneracy in neutrino oscillations, JHEP 01 (2010) 049 [arXiv:0904.3360] [INSPIRE].

[8] P. Coloma, A. Donini, J. Lopez-Pavon and H. Minakata, Non-standard interactions at a neutrino factory: correlations and CP-violation, JHEP 08 (2011) 036 [arXiv:1105.5936] [INSPIRE].

[9] T2K collaboration, Y. Itow et al., The JHF-Kamioka neutrino project, in Neutrino oscillations and their origin. Proceedings, $3^{\text {rd }}$ International Workshop, NOON 2001, Kashiwa Tokyo Japan December 5-8 2001 pg. 239 [hep-ex/0106019] [INSPIRE].

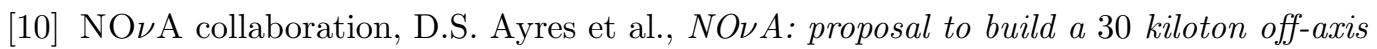
detector to study $\nu_{\mu} \rightarrow \nu_{e}$ oscillations in the NuMI beamline, [hep-ex/0503053] [INSPIRE].

[11] T2K collaboration, K. Abe et al., Sensitivity of the T2K accelerator-based neutrino experiment with an extended run to $20 \times 10^{21}$ POT, [arXiv: 1607.08004] [INSPIRE].

[12] T2K collaboration, K. Abe et al., Proposal for an extended run of T2K to $20 \times 10^{21}$ POT, [arXiv: 1609.04111] [INSPIRE].

[13] K. Abe et al., Letter of intent: the hyper-Kamiokande experiment - detector design and physics potential, [arXiv:1109.3262] [INSPIRE].

[14] K. Hagiwara, Physics prospects of future neutrino oscillation experiments in Asia, Nucl. Phys. Proc. Suppl. 137 (2004) 84 [hep-ph/0410229] [INSPIRE].

[15] M. Ishitsuka, T. Kajita, H. Minakata and H. Nunokawa, Resolving neutrino mass hierarchy and CP degeneracy by two identical detectors with different baselines, Phys. Rev. D 72 (2005) 033003 [hep-ph/0504026] [INSPIRE].

[16] K. Hagiwara, N. Okamura and K.-I. Senda, Solving the neutrino parameter degeneracy by measuring the T2K off-axis beam in Korea, Phys. Lett. B 637 (2006) 266 [Erratum ibid. B 641 (2006) 491] [hep-ph/0504061] [INSPIRE].

[17] K. Hagiwara, N. Okamura and K.-I. Senda, Physics potential of T2KK: an extension of the T2K neutrino oscillation experiment with a far detector in Korea, Phys. Rev. D 76 (2007) 093002 [hep-ph/0607255] [INSPIRE].

[18] T. Kajita, H. Minakata, S. Nakayama and H. Nunokawa, Resolving eight-fold neutrino parameter degeneracy by two identical detectors with different baselines, Phys. Rev. D 75 (2007) 013006 [hep-ph/0609286] [INSPIRE].

[19] K. Hagiwara and N. Okamura, Re-evaluation of the T2KK physics potential with simulations including backgrounds, JHEP 07 (2009) 031 [arXiv:0901.1517] [INSPIRE].

[20] K. Hagiwara, T. Kiwanami, N. Okamura and K.-I. Senda, Physics potential of neutrino oscillation experiment with a far detector in Oki Island along the T2K baseline, JHEP 06 (2013) 036 [arXiv:1209.2763] [INSPIRE].

[21] K. Hagiwara, P. Ko, N. Okamura and Y. Takaesu, Revisiting T2KK and T2KO physics potential and $\nu_{\mu}-\bar{\nu}_{\mu}$ beam ratio, [arXiv: 1605.02368] [INSPIRE].

[22] LBNE collaboration, C. Adams et al., The Long-Baseline Neutrino Experiment: exploring fundamental symmetries of the universe, [arXiv:1307.7335] [INSPIRE]. 
[23] DUNE collaboration, R. Acciarri et al., Long-Baseline Neutrino Facility (LBNF) and Deep Underground Neutrino Experiment (DUNE) conceptual design report volume 1: the LBNF and DUNE projects, [arXiv:1601.05471] [INSPIRE].

[24] DUNE collaboration, R. Acciarri et al., Long-Baseline Neutrino Facility (LBNF) and Deep Underground Neutrino Experiment (DUNE) conceptual design report volume 2: the physics program for DUNE at LBNF, [arXiv:1512.06148] [INSPIRE].

[25] S. Razzaque and A. Yu. Smirnov, Super-PINGU for measurement of the leptonic CP-phase with atmospheric neutrinos, JHEP 05 (2015) 139 [arXiv:1406.1407] [INSPIRE].

[26] J. Alonso et al., Expression of interest for a novel search for CP-violation in the neutrino sector: DAEdALUS, [arXiv:1006.0260] [INSPIRE].

[27] E. Ciuffoli, J. Evslin and F. Zhao, Neutrino physics with accelerator driven subcritical reactors, JHEP 01 (2016) 004 [arXiv:1509.03494] [INSPIRE].

[28] J. Cao et al., Muon-decay medium-baseline neutrino beam facility, Phys. Rev. ST Accel. Beams 17 (2014) 090101 [arXiv:1401.8125] [INSPIRE].

[29] M. Blennow, P. Coloma and E. Fernández-Martínez, The MOMENT to search for CP-violation, JHEP 03 (2016) 197 [arXiv: 1511.02859] [INSPIRE].

[30] E. Ciuffoli, J. Evslin and X. Zhang, The leptonic CP phase from muon decay at rest with two detectors, JHEP 12 (2014) 051 [arXiv:1401.3977] [INSPIRE].

[31] D.V. Forero and P. Huber, Hints for leptonic CP-violation or new physics?, Phys. Rev. Lett. 117 (2016) 031801 [arXiv:1601.03736] [INSPIRE].

[32] N. Klop and A. Palazzo, Imprints of CP-violation induced by sterile neutrinos in $T 2 K$ data, Phys. Rev. D 91 (2015) 073017 [arXiv:1412.7524] [inSPIRE].

[33] A. Palazzo, 3-flavor and 4-flavor implications of the latest T2K and NOvA electron (anti-)neutrino appearance results, Phys. Lett. B 757 (2016) 142 [arXiv:1509.03148] [INSPIRE].

[34] R. Gandhi, B. Kayser, M. Masud and S. Prakash, The impact of sterile neutrinos on CP measurements at long baselines, JHEP 11 (2015) 039 [arXiv:1508.06275] [INSPIRE].

[35] J. Liao, D. Marfatia and K. Whisnant, Degeneracies in long-baseline neutrino experiments from nonstandard interactions, Phys. Rev. D 93 (2016) 093016 [arXiv:1601.00927] [INSPIRE].

[36] M. Masud and P. Mehta, Nonstandard interactions spoiling the CP-violation sensitivity at DUNE and other long baseline experiments, Phys. Rev. D 94 (2016) 013014 [arXiv: 1603.01380] [INSPIRE].

[37] M. Masud, A. Chatterjee and P. Mehta, Probing CP-violation signal at DUNE in presence of non-standard neutrino interactions, J. Phys. G 43 (2016) 095005 [arXiv:1510.08261] [INSPIRE].

[38] A. de Gouvêa and K.J. Kelly, Non-standard neutrino interactions at DUNE, Nucl. Phys. B 908 (2016) 318 [arXiv:1511.05562] [inSPIRE].

[39] P. Coloma, Non-standard interactions in propagation at the Deep Underground Neutrino Experiment, JHEP 03 (2016) 016 [arXiv:1511.06357] [INSPIRE].

[40] P. Bakhti and Y. Farzan, CP-violation and non-standard interactions at the MOMENT, JHEP 07 (2016) 109 [arXiv: 1602.07099] [INSPIRE]. 
[41] A. de Gouvêa and K.J. Kelly, False signals of CP-invariance violation at DUNE, [arXiv: 1605.09376] [INSPIRE].

[42] M. Blennow, S. Choubey, T. Ohlsson, D. Pramanik and S.K. Raut, A combined study of source, detector and matter non-standard neutrino interactions at DUNE, JHEP 08 (2016) 090 [arXiv: 1606.08851] [INSPIRE].

[43] P. Bakhti and A.N. Khan, Sensitivities to charged-current nonstandard neutrino interactions at DUNE, [arXiv:1607.00065] [inSPIRE].

[44] J. Evslin, S.-F. Ge and K. Hagiwara, The leptonic CP phase from T2(H)K and $\mu^{+}$decay at rest, JHEP 02 (2016) 137 [arXiv: 1506. 05023] [INSPIRE].

[45] J. Kopp, M. Lindner, T. Ota and J. Sato, Non-standard neutrino interactions in reactor and superbeam experiments, Phys. Rev. D 77 (2008) 013007 [arXiv: 0708. 0152] [INSPIRE].

[46] T. Kikuchi, H. Minakata and S. Uchinami, Perturbation theory of neutrino oscillation with nonstandard neutrino interactions, JHEP 03 (2009) 114 [arXiv:0809.3312] [INSPIRE].

[47] A. De Rujula, M.B. Gavela and P. Hernández, Neutrino oscillation physics with a neutrino factory, Nucl. Phys. B 547 (1999) 21 [hep-ph/9811390] [INSPIRE].

[48] M. Freund, M. Lindner, S.T. Petcov and A. Romanino, Testing matter effects in very long baseline neutrino oscillation experiments, Nucl. Phys. B 578 (2000) 27 [hep-ph/9912457] [INSPIRE].

[49] E.K. Akhmedov, Matter effects in oscillations of neutrinos traveling short distances in matter, Phys. Lett. B 503 (2001) 133 [hep-ph/0011136] [INSPIRE].

[50] P. Lipari, CP violation effects and high-energy neutrinos, Phys. Rev. D 64 (2001) 033002 [hep-ph/0102046] [INSPIRE].

[51] H. Minakata and H. Nunokawa, Measuring leptonic CP-violation by low-energy neutrino oscillation experiments, Phys. Lett. B 495 (2000) 369 [hep-ph/0004114] [INSPIRE].

[52] H. Minakata, The three neutrino scenario, Nucl. Phys. Proc. Suppl. 100 (2001) 237 [hep-ph/0101231] [INSPIRE].

[53] O. Yasuda, Vacuum mimicking phenomena in neutrino oscillations, Phys. Lett. B 516 (2001) 111 [hep-ph/0106232] [INSPIRE].

[54] O.L.G. Peres and A. Yu. Smirnov, Atmospheric neutrinos: LMA oscillations, $U_{e 3}$ induced interference and CP-violation, Nucl. Phys. B 680 (2004) 479 [hep-ph/0309312] [InSPIRE].

[55] M. Blennow and A. Yu. Smirnov, Neutrino propagation in matter, Adv. High Energy Phys. 2013 (2013) 972485 [arXiv:1306.2903] [INSPIRE].

[56] E.K. Akhmedov, A. Dighe, P. Lipari and A.Y. Smirnov, Atmospheric neutrinos at Super-Kamiokande and parametric resonance in neutrino oscillations, Nucl. Phys. B 542 (1999) 3 [hep-ph/9808270] [INSPIRE].

[57] H. Yokomakura, K. Kimura and A. Takamura, Overall feature of CP dependence for neutrino oscillation probability in arbitrary matter profile, Phys. Lett. B 544 (2002) 286 [hep-ph/0207174] [INSPIRE].

[58] S.-F. Ge, K. Hagiwara and C. Rott, A novel approach to study atmospheric neutrino oscillation, JHEP 06 (2014) 150 [arXiv:1309.3176] [INSPIRE].

[59] T. Wongjirad, Opportunities with decay-at-rest sources, in Neutrino, (2016). 
[60] Particle Data Group collaboration, J. Beringer et al., Review of particle physics (RPP), Phys. Rev. D 86 (2012) 010001 [InSPIRE].

[61] K. Asano and H. Minakata, Large- $\theta_{13}$ perturbation theory of neutrino oscillation for long-baseline experiments, JHEP 06 (2011) 022 [arXiv:1103.4387] [INSPIRE].

[62] X.-J. Xu, Why is the neutrino oscillation formula expanded in $\Delta m_{21}^{2} / \Delta m_{31}^{2}$ still accurate near the solar resonance in matter?, JHEP 10 (2015) 090 [arXiv: 1502.02503] [INSPIRE].

[63] M.C. Gonzalez-Garcia and M. Maltoni, Determination of matter potential from global analysis of neutrino oscillation data, JHEP 09 (2013) 152 [arXiv:1307.3092] [INSPIRE].

[64] S.-F. Ge, P. Pasquini, M. Tortola and J.W.F. Valle, Measuring the leptonic CP phase in neutrino oscillations with non-unitary mixing, [arXiv:1605.01670] [INSPIRE].

[65] S.-F. Ge, NuPro: a simulation package for neutrino properties webpage, http://nupro.hepforge.org/.

[66] T2K collaboration, K. Abe et al., Neutrino oscillation physics potential of the T2K experiment, Prog. Theor. Exp. Phys. 2015 (2015) 043C01 [arXiv:1409.7469] [InSPIRE]. 